\title{
Searching for transits in the Wide Field Camera Transit Survey with difference-imaging light curves
}

\author{
J. Zendejas Dominguez ${ }^{1,2}$, J. Koppenhoefer ${ }^{2,1}$, R. P. Saglia ${ }^{2,1}$, J. L. Birkby ${ }^{3}$, S. T. Hodgkin ${ }^{4}$, G. Kovács ${ }^{4}$, \\ D. J. Pinfield ${ }^{5}$, B. Sipőcz ${ }^{5}$, D. Barrado ${ }^{6,7}$, R. Bender ${ }^{2,1}$, C. del Burgo ${ }^{8}$, M. Cappetta ${ }^{2}$, E. L. Martín ${ }^{9}$, S. V. Nefs ${ }^{3}$, \\ A. Riffeser ${ }^{1}$, and P. Steele ${ }^{2}$ \\ 1 University Observatory Munich, Scheinerstrasse 1, 81679 München, Germany \\ e-mail: chicho@usm.uni-muenchen.de \\ 2 Max Planck Institute for Extraterrestrial Physics, Giessenbachstrasse 1, 85748 Garching, Germany \\ 3 Leiden Observatory, Leiden University, Postbus 9513, 2300 RA Leiden, The Netherlands \\ ${ }^{4}$ Institute of Astronomy, Cambridge University, Madingley Road, Cambridge CB3 OHA, UK \\ ${ }^{5}$ University of Hertfordshire, Centre for Astrophysics Research, Science and Technology Research Institute, College Lane, \\ AL10 9AB Hatfield, UK \\ ${ }^{6}$ Depto. Astrofísica, Centro de Astrobiología (INTA-CSIC), ESAC campus, PO Box 78, 28691 Villanueva de la Cañada, Spain \\ 7 Calar Alto Observatory, Centro Astronómico Hispano-Alemán, C/ Jesús Durbán Remón, 04004 Almería, Spain \\ 8 Instituto Nacional de Astrofísica, Óptica y Electrónica, Luis Enrique Erro 1, Sta. Ma. Tonantzintla, 72840 Puebla, Mexico \\ 9 Centro de Astrobiología (CSIC-INTA), Ctra. Ajalvir km. 4, 28850 Torrejón de Ardoz, Madrid, Spain
}

Received 18 February 2013 / Accepted 2 October 2013

\section{ABSTRACT}

\begin{abstract}
The Wide Field Camera Transit Survey is a pioneer program aiming at for searching extra-solar planets in the near-infrared. The images from the survey are processed by a data reduction pipeline, which uses aperture photometry to construct the light curves. We produce an alternative set of light curves using the difference-imaging method for the most complete field in the survey and carry out a quantitative comparison between the photometric precision achieved with both methods. The results show that differencephotometry light curves present an important improvement for stars with $J>16$. We report an implementation on the box-fitting transit detection algorithm, which performs a trapezoid-fit to the folded light curve, providing more accurate results than the boxfitting model. We describe and optimize a set of selection criteria to search for transit candidates, including the $V$-shape parameter calculated by our detection algorithm. The optimized selection criteria are applied to the aperture photometry and difference-imaging light curves, resulting in the automatic detection of the best 200 transit candidates from a sample of $\sim 475000$ sources. We carry out a detailed analysis in the 18 best detections and classify them as transiting planet and eclipsing binary candidates. We present one planet candidate orbiting a late G-type star. No planet candidate around M-stars has been found, confirming the null detection hypothesis and upper limits on the occurrence rate of short-period giant planets around M-dwarfs presented in a prior study. We extend the search for transiting planets to stars with $J \leq 18$, which enables us to set a stricter upper limit of $1.1 \%$. Furthermore, we present the detection of five faint extremely-short period eclipsing binaries and three M-dwarf/M-dwarf binary candidates. The detections demonstrate the benefits of using the difference-imaging light curves, especially when going to fainter magnitudes.
\end{abstract}

Key words. planets and satellites: detection - methods: data analysis - techniques: photometric

\section{Introduction}

In recent years, the search for exo-planets has become an interesting and exciting field in astronomy. About one thousand exoplanets have been found since Mayor \& Queloz (1995) detected the first planet orbiting its host main sequence star. Measuring the host-star radial-velocity variations represents one of the most successful techniques to detect exo-planets; nevertheless, only few parameters of the planetary system can be determined with this method. This changes if we search for a planet transiting to its host companion. A transit occurs when a planet blocks part of the surface from the star causing a slight and periodic variation in its brightness, which can be detected by a photometric analysis. This analysis provides information of the planet and its host star, which can be used along with radial velocity measurements to deduce important physical parameters of the transiting planet, such as the mass and the radius. The first planetary transit signal was reported in 2000 (Charbonneau et al. 2000; Henry et al. 2000), and since this discovery, a significant number (more than 300) of exo-planets have been detected transiting their host star.

Recently, several transit missions and surveys have been designed to find and characterize new exo-planets. The most exciting and successful projects designed to detect periodic transits are the space missions Kepler (Borucki et al. 2010) and CoRoT ${ }^{1}$ (Aigrain et al. 2008; Barge et al. 2008). Kepler was launched on March 6, 2009 to observe more than 150000 stars, and it is expected to find a large number of Earth-size planets and superEarths. On the other hand CoRoT was originally designed to find exo-planets with properties comparable to rocky planets in our solar system. Nevertheless, the culmination of CoRoT was announced in June 2013 after six years of successful operation.

Earth-like planets are particularly interesting: if they revolve in the habitable zone of their host star (Kasting et al. 1993), the environment may be adequate to support liquid water on the surface of the planet, which is believed to be a key for

Convection, Rotation and Planetary Transits. 
the development of life. Cool and low-mass M-dwarf stars are the most promising candidates to find Earth-like planets and super-Earths. Due to their low effective temperature $\left(T_{\text {eff }}\right)$, the change in their brightness caused by a planet orbiting around them is more evident. For instance, an Earth-size planet orbiting a $0.08 M_{\odot}$ star produces a transit of $1 \%$ depth (Kaltenegger \& Traub 2009); a similar effect occurs when a Jupiter-size planet blocks a Sun-like star.

Searching for transiting planets at near-infrared (NIR) wavelengths provides important advantages to detect transiting planets around M-dwarfs, since the peak of the spectral energy distribution (SED) of these stars falls in this spectral range. Several projects are dedicated to study transiting planets around M-dwarf, such as APACHE (Giacobbe et al. 2012), PTF/Mdwarfs (Law et al. 2012) and TRAPPIST (Jehin et al. 2011). However, there are so far only two transit projects that are focused on finding exo-planets around cool and low-mass stars at NIR wavelengths, namely the MEarth project and the Wide Field Camera (WFCAM) ${ }^{2}$ transit survey (WTS). The MEarth project (Irwin et al. 2009; Berta et al. 2012) is a transit survey that operates since 2008 with eight independent $0.4 \mathrm{~m}$ robotic telescopes located at the Fred Lawrence Whipple Observatory on Mount Hopkins, Arizona, and is soon expected to include eight additional telescopes in the southern hemisphere. The survey individually monitors $\sim 2000$ nearby $(<33$ pc) M-dwarfs in the NIR and is designed to detect exo-planets as small as $2 R_{\oplus}$. On the other hand, the WTS is a pioneer project operated since 2007 with observations from the United Kingdom Infrared Telescope (UKIRT) that stands out for its particular aims and methodology. A brief description of the WTS is summarized in Sect. 2.1.

Traditionally, aperture photometry (AP) has been the standard technique to produce light curves in transit surveys. In 1996, a new method to study crowded fields by optimal image subtraction was presented by Tomaney \& Crotts (1996) and subsequently improved by Alard \& Lupton (1998). This method (usually called difference-imaging DI) was initially developed to study microlensing events in crowded fields. However, image subtraction photometry has become an important tool for searching for planetary transits (Pietrukowicz et al. 2010), since the majority of transit survey targets are crowded fields (e.g. Galactic plane). In the past, some authors have carried out comparisons between different photometric techniques. For instance, Montalto et al. (2007) used the data from a ten-night observing campaign from four different ground-based telescopes to develop a quantitative test by comparing the photometric precision of three different photometry algorithms: AP, point spread function (PSF)-fitting photometry, and image subtraction photometry. They compare the photometric precision as a function of the apparent visual magnitude for all photometric techniques. Due to the several factors involved in the observations (which influence directly in the measurement), such as size of the telescope, instruments, or atmospheric conditions, the quality of the light curves clearly varies depending on the location of the observations. For all cases presented in Montalto et al. (2007), the best root mean square (rms) value was achieved by image subtraction photometry; in some cases, a difference of up to 4 mmag is observed for bright objects. On the other hand, AP and PSF-fitting photometry show significant variations of the photometric precision, as achieved by each telescope. This discrepancy suggests that the precision obtained by a certain photometric technique

\footnotetext{
2 Wide Field Camera, see http://www. jach.hawaii.edu/UKIRT/ instruments/wfcam/
}

may depend on the characteristics of the survey; that is, a particular method might produce different results depending on the observing conditions. In this work, we carry out a similar analysis by comparing the photometric precision of the WTS light curves obtained by DI and AP.

Large sets of light curves usually show systematic effects that can be associated with the atmospheric extinction, detector efficiency, or PSF changes on the detector. The sysrem algorithm proposed by Tamuz et al. (2005) has been widely tested and it is commonly used in transit surveys (Snellen et al. 2007; Pont et al. 2006) to decrease the number of systematics in light curves. To reduce these effects in our sample, we apply the sysrem algorithm and subsequently include the results in the comparison analysis.

Due to the large number of light curves in transit surveys, an efficient detection algorithm is needed to speed up the identification of planet candidates. Several algorithms have been developed after the discovery of the first planet transiting its host star. For instance, Defaÿ et al. (2001) presented an algorithm that uses a multi-frequency Fourier fit to model periodic astronomical time series. Kovács et al. (2002) presented a box-fitting algorithm based on least squares fit of step functions (BLS) to analyze stellar photometric time series. This algorithm has shown significantly better results than previous works, and it has become a popular tool for searching for exo-planets in transit surveys. Recently, the Transit Planet Search (TPS) algorithm (Jenkins et al. 2010) has been developed to be part of the Kepler science processing pipeline to search for transit planets, which is able to achieve super-resolution detection statistics.

False positives and false detections are common problems that make difficult the search for exo-planets in transit surveys. A false detection can be caused if the light curve holds a significant number of systematic outliers, which can produce fake signals, whereas a false positive is associated to real variability of the flux from the host star (e.g., eclipsing binary systems or intrinsic variable stars). Although false positives and false detections have conceptually different origins, both scenarios are referred as false positives for practical reasons in this work. Nowadays, large scale transit surveys require strategies to efficiently weed out false positives in candidate samples and reduce the number of light curves inspected by visual examination. Several authors have suggested methods to reduce the number of false positives and facilitate the selection of the best candidates. For instance, Burke et al. (2006) proposed a series of selection criteria based on a $\chi^{2}$-minimization equivalent to the analytic solutions provided by BLS method. Later on, Hartman et al. (2009) presented selection criteria divided in different steps, which include the signal-to-pink noise ratio (Pont et al. 2006), the number of data points in the light curves, a magnitude limit, and exclusion of sources with alias periods between 0.99 and 1.02 days or less than 0.4 days. Nevertheless, the majority of selection criteria only remove false positives that are not related to real astrophysical variability. In this study, we propose a set of selection criteria with the ability of excluding false positives by considering elements from the transit detection algorithm, including a new criterion named the $V$-shape parameter, which is designed to automatically recognize eclipsing binary systems.

The structure of this paper follows the next outline: in Sect. 2, we describe the WTS and summarize the image reduction pipeline. In this section, we also give a description of the DI analysis and describe the procedure of the light curve extraction. A quantitative comparison between the photometric precision of light curves obtained by AP and DI techniques is presented in Sect. 3. Section 4 is dedicated to present our transit-detection 
algorithm and the $V$-shape parameter as obtained from the implementations made on the BLS algorithm. Sections 4.1 and 4.2 present our selection criteria and the optimization of the parameters used to detect planet candidates on the WTS light curves. In Sects. 5 and 6, we show the candidates that pass our selection criteria and a detailed physical characterization of the candidates. We present other applications of the WTS DI light curves in Sect. 7, such as the detection of ultra-short period M-dwarf binaries and detached M-dwarf eclipsing binaries. Finally, we summarize our results in Sect. 8.

\section{Data analysis and methodology}

\subsection{The Wide Field Camera Transit Survey}

Low-mass main-sequence stars of spectral type $M$ are the most abundant stars in the Galaxy, representing about $75 \%$ of the total stellar population (Scalo et al. 2007). In addition, M-dwarfs present certain properties that make them ideal targets to search for rocky planets (Tarter et al. 2007). Motivated by this, the WTS was initially developed to monitor and search for transiting planets for the first time in the NIR. Since the transit technique is based on relative photometry, the survey can be performed in poor weather conditions; hence, WTS is conducted as a back-up project, operating when the observing conditions are not suitable (seeing $>1$ arcsec) for the main program of the UKIRT Infrared Deep Sky Survey (UKIDSS). The survey was originally assigned to about 200 nights at the $3.8 \mathrm{~m}$ UKIRT, which is equipped with the WFCAM and consists of four Rockwell Hawaii-II arrays with $2048 \times 2048$ pixels in each panel that cover a field of view of $\sim 0.19$ square degrees with a resolution of $0.4 \mathrm{arcsec} /$ pixels. The four detectors are distributed geometrically at the corners of a square with an auto-guider located at the center of the frame. This array is usually called pawprint. A complete observation sequence of the WTS consists of 8 pawprints (a-h), and each one is built up from a nine point jitter pattern of $10 \mathrm{~s}$. An entire field is completed in about $15 \mathrm{~min}$. That is, the WTS light curves have an average cadence of four data points per hour. Since the dimension and separation of the detectors have approximately the same size ( 13 arcmin), a uniform target field can be achieved by observing the 8 pawprints. Four fields were selected seasonally to be observed ( $\mathrm{RA}=03,07,17$, and $19 \mathrm{~h}$ ) periodically during a year; thereby, the WTS guarantees a reasonable continuous observations campaign. Nevertheless, this work is only dedicated to study the RA $=19 \mathrm{~h}$ field, which has been observed until May 2011 with about 1145 epochs. It contains $\sim 475000$ sources, of which $\sim 113000$ have magnitudes $J \leq 18$. All observations for the WTS are done in the $J$-band $\left(\lambda_{\text {eff }} \approx 1200 \mathrm{~nm}\right)$. For more details about the WTS, we refer to Kovács et al. (2013). The image reduction procedure is described in the next section.

\subsection{Image reduction pipeline}

Due to the large amount of data collected by the WTS, a pipeline to process the images automatically is required. The $J$-band images from the WTS are reduced by the image reduction pipeline from the Cambridge Astronomical Survey Unit ${ }^{3}$ (CASU), which is used to process all images from the WFCAM. The image reduction pipeline is based on the work developed by Irwin (1985) and was later modified and adapted to the Isaac Newton Telescope (INT) Wide Field Survey (WFS; Irwin \& Lewis 2001), and subsequently, to the Monitor project

\footnotetext{
3 http://casu.ast.cam.ac.uk/surveys-projets/wfcam
}

(Irwin et al. 2007). The pipeline includes the following steps: de-biassing and trimming, non-linearity correction, bad pixel replacement, flatfielding, defringing, and sky subtraction. A thorough description of all the steps can be found in Irwin \& Lewis (2001). Astrometry and photometry are calibrated using bright stars in the field-of-view from the 2-Micron All-Sky Survey (2MASS; Kleinmann et al. 1994) catalog (see Hodgkin et al. 2009). Particularly, the astrometric calibration plays an important role in the DI technique, since a precise alignment of data frames is crucial to success with this method. The astrometry is described by six coefficient linear transformations that allow for scale, rotation, shear and coordinate offset corrections. The pipeline also provides master catalog and light curves, which are constructed by the AP method, using a series of soft-edge apertures that account for the fractional area of a pixel included in the aperture and a simultaneous redistribution of flux from nearby stars. More detailed descriptions of the light curves and catalog can be found in Kovács et al. (2013). In the next section, we describe the DI method and the process of the light curves extraction.

\subsection{Difference-imaging analysis}

In addition to the standard WTS light curves (AP) generated by the CASU pipeline, we alternatively produce a second set of light curves by using DI photometry. According to Alard \& Lupton (1998), the method operates on a reference image, which is the combination of the best seeing images from the data set ( $\sim 20$ in our case). On average, the seeing range of the images used to construct the reference frames is 1.18 to 1.39 arcsec. The reference frame is convolved with a kernel to match the seeing of each single image, resulting in a convolved reference image. A difference image is obtained by subtracting the convolved reference image from each single image.

Finding the optimal kernel that matches the seeing of two frames with different PSFs represents a crucial and complex problem during the DI process. Alard \& Lupton (1998) proposed a method, in which the optimal kernel is approximated using a superposition of $N$-kernel base functions, which constitute 2-dimensional Gaussian functions modulated with a polynomial of order $p_{i}$. The expression for the optimal kernel is

$K(u, v)=\sum_{i=1}^{N} \exp \left[-\frac{u^{2}+v^{2}}{2 \sigma_{i}^{2}}\right] \sum_{j=0}^{p_{i}} \sum_{k=0}^{p_{i}-j} a_{i j k} u^{j} v^{k}$

where $u$ and $v$ are the pixel coordinates of the kernel bitmap, which has the same pixel size as the images; $a_{i j k}$ are the coefficients from the decomposition of the kernel using basis of functions; and $\sigma_{i}$ is the variance related to the Gaussian distribution. To calculate the kernel, we use four base functions $(N=4)$ with $\sigma_{i}=1,2,3$ and 0.1 , while the degrees of the associated polynomials $p_{i}$ are $6,4,2$ and 0 , respectively. The kernel size is $11 \times 11$ pixels, and we consider a 1 st order background polynomial to account for background difference. All free parameters, such as the $a_{i j k}$ coefficients and the parameters associated with the background polynomial, are determined by minimization of the following expression,

$\chi^{2}=\sum_{x, y} \frac{1}{\sigma_{x, y}^{2}}[\{R(x, y) \otimes K(u, v)\}+B(x, y)-S(x, y)]^{2}$,

where $\sigma_{x, y}^{2}$ is the variance of a Gaussian distribution used to approximate the Poisson image statistics, $S(x, y)$ is a single image, $R(x, y)$ is the reference frame, and $B(x, y)$ is the polynomial surface function that accounts for background differences. 
Variations of PSF over the detector are a common problem in the DI technique. To reduce this effect during the estimation of the kernel, we divide the images in subfields and calculate the kernel in each subfield. In our case, we divide the images in $10 \times 10$ subfields with a size of $200 \times 200$ pixels.

To achieve an optimized set of difference images, we tested several parameters. For each set, we extract the light curves and measure the photometric precision to verify the quality of the sample. During the testing process, we found that the light curve precision is significantly improved if we mask bright or faint stars, while the difference images are produced. Two sets of difference images are created to guarantee the best quality of the light curves. In a first set, we mask all sources with magnitudes $J \leq 16$, which provides an improvement for objects fainter than this threshold. The second set is processed by masking faint objects; that is, all sources that hold magnitudes $J>16$, which results in an improvement for bright stars.

\subsection{Light curve extraction}

From the difference images, we are able to measure the differential flux of each source. Adding the value measured in the reference image, the total flux for each single star can be estimated. Although differential fluxes are relative easy to measure in the difference images because all constant sources are removed, estimating the fluxes in the reference frame is more difficult, especially for objects that have close neighbors. We measure the flux in the reference frame using iterative PSF-photometry. This technique is very successful to measure flux accurately in crowded fields. The method uses bright and isolated stars to extract the PSF. In a first step, an initial estimation of the flux of each star is measured from the extracted PSF. In subsequent iterations, all nearby stars are removed before measuring the flux of a particular source. This process continues until all fluxes converge, using the improved flux measured in the previous step in each iteration. The fluxes measured in difference images are also estimated by PSF-photometry. The PSF is obtained from the convolved reference image by using the same stars that are employed to estimate the flux in the reference frame, which are a representative sample of stars in each field. Although the fluxes in the difference images certainly could be estimated by using a different photometric technique (e.g. aperture photometry) since the stellar crowd in the field is eliminated, we have chosen PSF-photometry to measure the fluxes because this method is not affected by dead pixels and does not require aperture corrections, which might lead to a wrong evaluation of the flux. Finally, the light curves are normalized to one and barycentrically time-corrected using the formula of Meeus (1982). The process of extracting the light curves is applied to both sets (one optimized for bright sources and one optimized for faint sources, see previous section). We obtain the optimized set by choosing the light curve with the better photometric precision for each source.

\section{Quality of the difference-imaging light curves and comparison with the aperture photometry method}

In this section, we compare the quality of the WTS light curves produced by AP (from the CASU pipeline) and DI. A quantitative comparison between the photometric precision of both sets of light curves is performed by calculating the rms of each single light curve from the two photometric analysis. During this process, we clip all $4 \sigma$ outliers, while clipping 3 and $5 \sigma$ outliers, provides similar results. Note that this step is only for the purpose of calculating the rms and is not a final operation on the light curves.

\subsection{Sysrem algorithm}

An algorithm to remove systematic effects in large sets of light curves from photometric surveys was proposed by Tamuz et al. (2005). The algorithm, called sysrem, has shown the capability of considerably improving the photometric precision of the data set by removing systematics related to the detector efficiency, PSF variations over the detector, or effects associated with the atmospheric extinction (Mazeh et al. 2009; Irwin et al. 2007). The algorithm searches for systematics that consistently appear in many sources of the sample; hence, sysrem has the ability to remove effects without any prior knowledge of the origin of the effect.

To improve the quality of the light curves, we consistently apply the sysrem algorithm to DI and AP light curves. Note that Irwin et al. (2007) showed that the sysrem algorithm does not improve the precision of AP light curves by much, and it might additionally produce false variability from the residuals. In our case, we find a significant reduction of the scatter of constant light curves for both DI and AP light curves. Any possible false variability created by sysrem does not lead to the detection of false positive candidates, since we use conservative criteria in the candidate selection process (see below).

The results are shown in Fig. 1, which represent the rms of the DI and AP light curves (panels a and b, respectively) as a function of the WFCAM $J$-band magnitude, after applying the sysrem algorithm. The DI light curves reach a precision of $3.5 \mathrm{mmag}$ for bright objects in the range of $12<J<14$, while the rms of AP light curves that are corrected by the sysrem algorithm reaches a precision of $\sim 2.5 \mathrm{mmag}$ in the same $J$-band magnitude interval. The plots show that DI produces better results for faint objects $(J>16)$; however the quality of AP light curves is slightly better in the bright magnitude range. For magnitudes larger than $J=16$, the DI light curves show a much higher photometric precision than the AP light curves. The rms shows presents a difference up to $5 \mathrm{mmag}$ at $J=17 \mathrm{mag}$ and 15-20 mmag at $J=18 \mathrm{mmag}$.

These results contrast to previous studies, which compare the photometric precision achieved with both methods. For example, Montalto et al. (2007) show that DI photometry achieves an equal or better photometric precision as compared to aperture and PSF photometry for all magnitudes. However, these studies were done at optical wavelengths ( $V$-band) and a direct comparison to a NIR survey (like the WTS) is not possible, since the detector characteristics are different. Imperfect treatment of nonlinearity effects at the bright end could be one possible source for the additional systematic noise that we observe in our DI light curves. Another problem might be the non-homogeneous background, which is visible in the WTS images. We can rule out that the effect is caused by a low astrometric accuracy. Any shifts between the reference frame and the single images would produce dipole-shaped residuals in the difference image; contrary to this effect, bright sources show very symmetric noise residuals in our difference images.

To show the capability of the sysrem algorithm to improve the photometric precision, we perform a similar quantitative analysis (see above) on the DI light curves by comparing the rms of the light curves before and after applying the sysrem algorithm. Figure 2 shows the rms difference between both sets of light curves as a function of the $J$-band magnitude. The result 


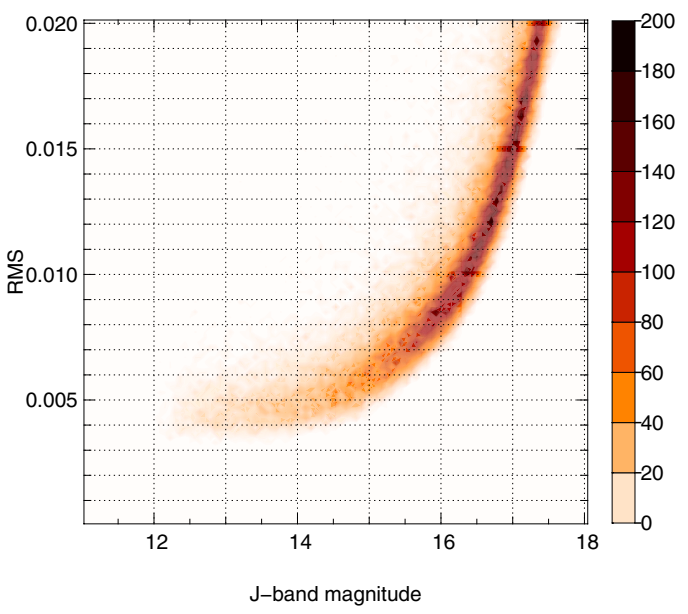

(a)

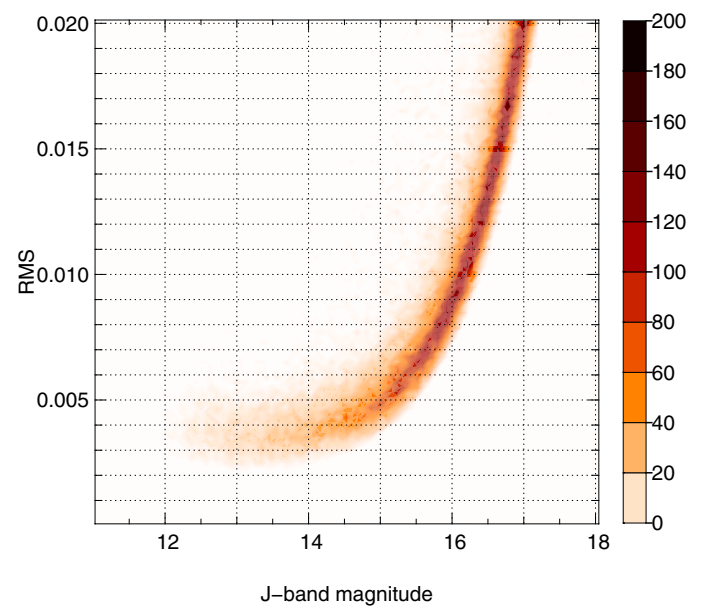

(b)

Fig. 1. Quantitative comparison between different photometric analyses. The figure shows the rms of the DI and AP light curves (panels a) and b), respectively) as a function of the $J$-band magnitude. The rms corresponds to the measurements obtained after removing systematic effects. The plot is displayed in density of data points in a scale of 100 bins.

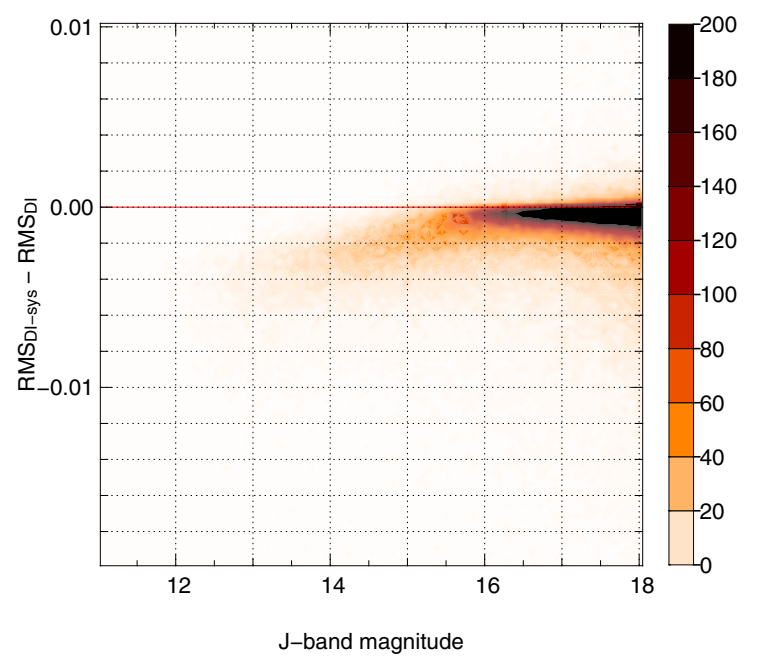

Fig. 2. rms difference between the DI light curves before and after applying sysrem algorithm. The plot shows the improvement achieved in the photometric precision once systematic effects are corrected. The plot shows the density of data points distributed in 100 bins.

of the comparative analysis indicates a significant improvement in the photometric precision of bright and faint sources when the sysrem algorithm is employed. A similar result is observed in the AP light curves. Although applying the sysrem algorithm results in an improvement of the photometric precision of the light curves, the capability of the algorithm to remove systematics effects is limited. Figure 3 shows the number of detected periods around the one-day alias period before and after using the sysrem algorithm. In an ideal case, the algorithm should account for these effects and eliminate the alias peak. In our case, the number around the alias period is reduced by a factor $\sim 2$ after applying the sysrem algorithm.

\subsection{Correction of the point-by-point errors derived from the individual images}

After removing systematic effects, we carried out a routine visual inspection over a random sample of light curves. We noticed

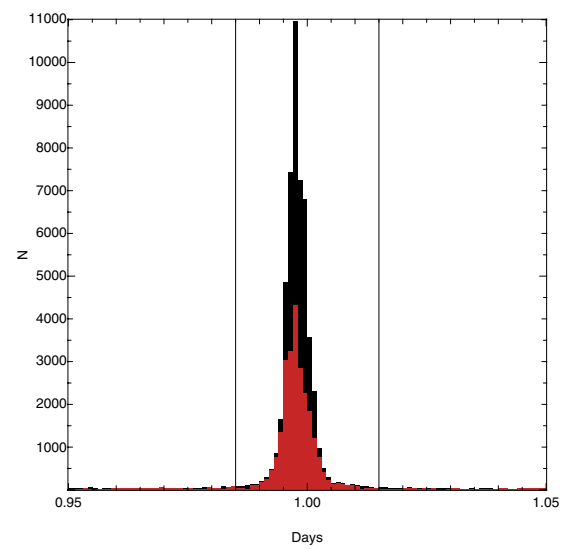

Fig. 3. Histogram of periods found by our transit detection algorithm, before (black) and after (red) applying the sysrem algorithm. The numbers of false positives between 0.985 and 1.015 day periods is reduced by a factor of 2 .

that the scatter of the data points was much larger than the error bars for many light curves (typically for bright sources). Usually error bars of light curves are estimated by a pipeline that takes different factors in account, such as the photon noise of the source, background contribution, and read-out noise. However, systematic effects caused by PSF-variations or variation in noise level from the background are not included. This seems to be the case of the WTS light curves, which present a wrong estimation of the error bars, which is correlated to the brightness of the object. A simple way to correct the size of the error bars is to compare the rms of the photometric measurements with the error values, since the rms is related to the scatter level and can be associated with the real error for non-variable objects. We perform this test for DI and AP light curves by dividing the rms by the median error calculated in each light curve. The results are shown in Fig. 4, where this quotient is plotted as a function of the $J$-band magnitude. If the error values were correct, the rms and median error should present similar values; therefore, data points in the plots should be distributed around 1. Nevertheless, there is an evident discrepancy between both quantities, which is reflected in the shape of the data point distributions. For our 


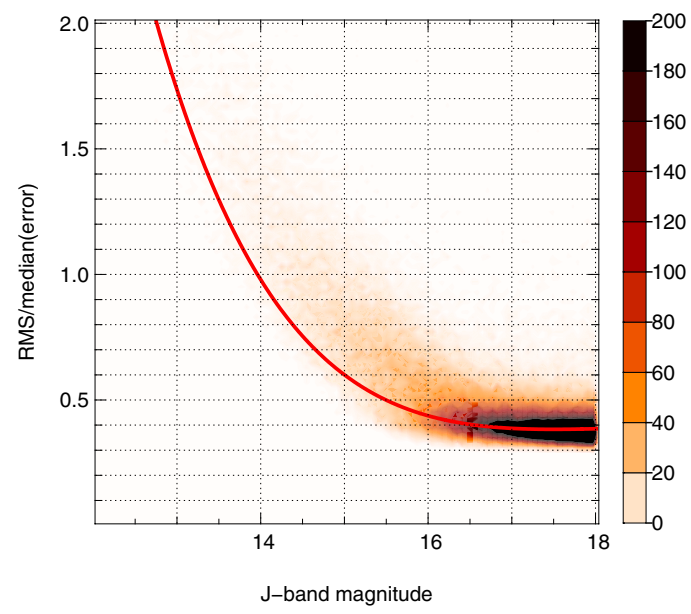

(a)

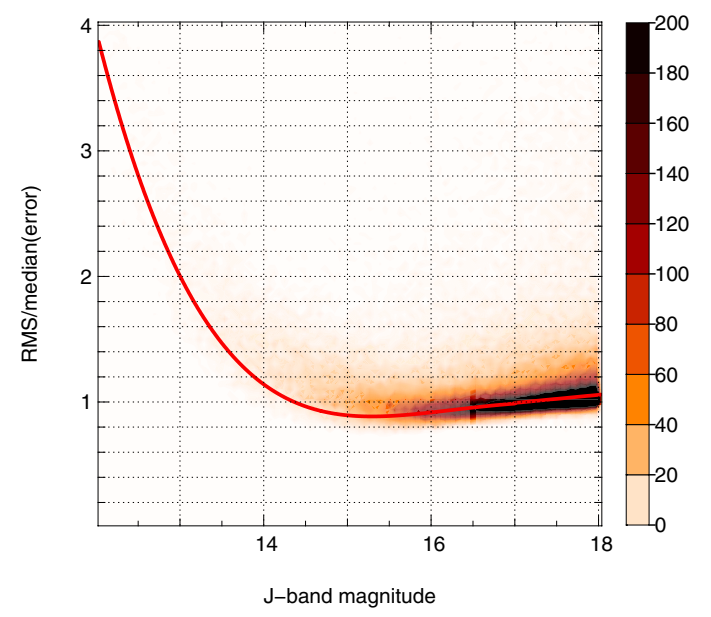

(b)

Fig. 4. Data point distribution of the rms divided by the median error from DI and AP light curves (panels a) and b) respectively) as a function of $J$-band magnitude. The solid-red line represents the polynomial used to scale the errors bars. The plot is displayed in density of data points in a scale of 100 bins.

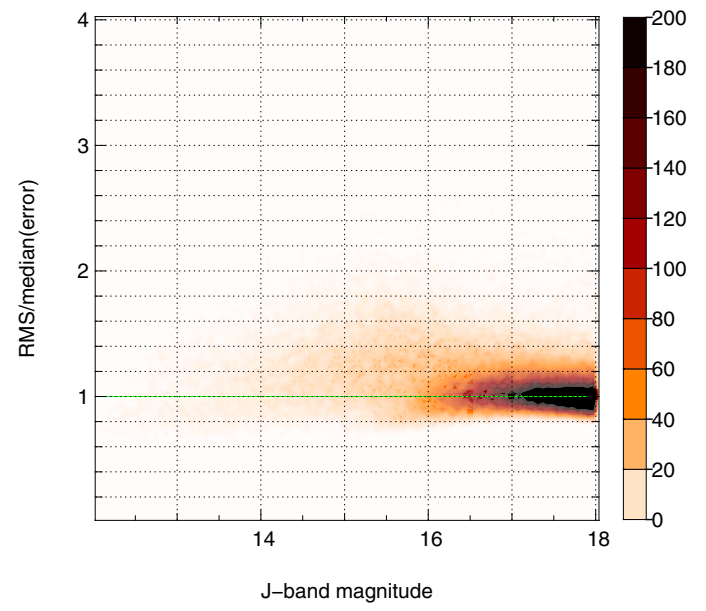

(a)

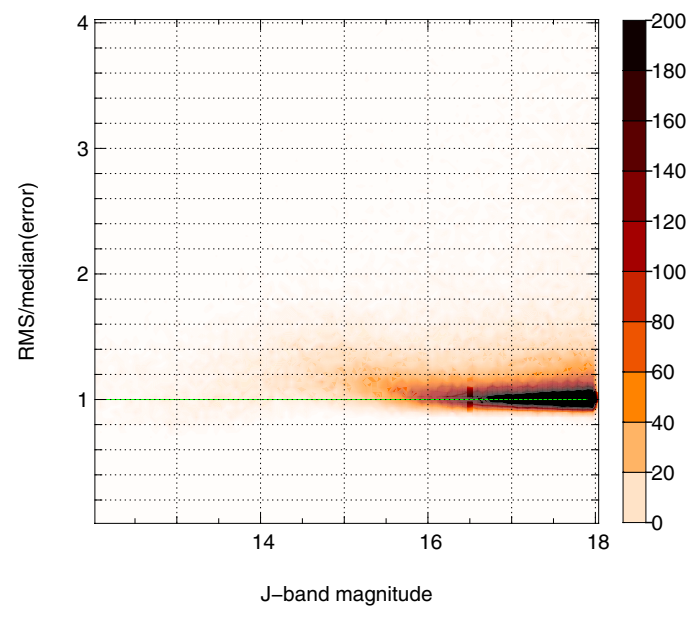

(b)

Fig. 5. Distribution of data points of the quotient rms/median(error) after re-scaling the error bars on the a) DI and b) AP light curves.

work, it is important to correct the bad estimation on the error bars, since some of our selection criteria (see below) and several parameters that we estimate for our candidates later on depend on the error bars. To correct the error bars, we fit a polynomial (Fig. 4) to the distribution of data points. The polynomial provides a scale factor as a function of the magnitude, which can be used to correct the whole sample. Unlike replacing the errors obtained from the images by an estimation of the scatter (rms), we avoid introducing an overestimation of the errors by scaling the error bars with a factor that is a function of the brightness of the objects, principally for variable sources, which can present a significant scatter in the light curves. After scaling the error bars, we perform the same test and show the results in Fig. 5, where we can see that the distribution of data points clearly has been adjusted and is now located close to 1 . Nevertheless, these figures present a second higher rms sequence for bright stars (14-16 mag). We know from the AP light curves (see Kovács et al. 2013) that the WTS data present a high level of red noise (Pont et al. 2006), which is also correlated to the magnitude of the objects, with the bright sources being the most affected for this effect. Although the sysrem algorithm is designed to filter out the red noise, there is a component from the red/pink-noise that remains in the sample of light curves, which can be observed in Fig. 4, where a significant scatter is visible in the distribution of the data points. Because the sysrem algorithm cannot eliminate completely this component of the $\mathrm{red} /$ pink noise, fake signals and a subsequent large number of false positives may be produced. Figure 3 demonstrates that the remaining systematics produce such effects, since a large number of objects fall into the daily alias. Nevertheless, the selection criteria that are used to detect planet candidates in the WTS light curves (see Sect. 4.1) have the capability to provide a pure candidate sample, ruling out false positives related to some of these systematics. On the other hand, we do not use the correlated noise to measure the transit-fitting significance in this work. Therefore, the polynomial used to correct the error bars does not consider the dispersion of data points generated by the remaining red/pink-noise component.

\section{Light curve analysis and transit detections}

We detect transits in the WTS light curves using an algorithm that is based on the BLS algorithm proposed by Kovács et al. (2002). Our modifications include a trapezoidal re fit of the 


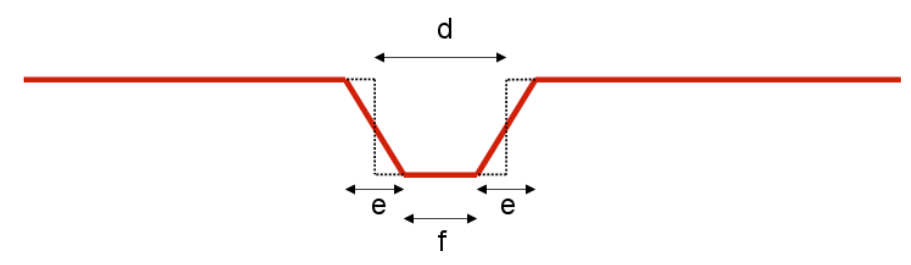

Fig. 6. Geometry of the symmetrical trapezoid-fit.

box-shaped eclipse found by BLS, where the re fit is done by symmetrically varying the edges of the box, while keeping the duration of the eclipse (" $d$ ") fixed, which is measured at half the transit depth (see Fig. 6). We emphasize that the trapezoidal shape is only fitted after the standard parameters provided by the box-fitting algorithm have been found (such as period, transit duration and epoch); however, the eclipse depth may change. We introduce the $V$-shape parameter:

$V=\frac{2 e}{f+2 e}$

where $e$ is the duration of the ingress/egress of the eclipse in phase units, and $f$ is the duration of the transit, or flat part of the trapezoid shape (see Fig. 6). If $e$ is considerably smaller than $f$, the $V$-shape parameter is close to 0 and the shape of the fit is box-like. On the other hand, if $f \approx 0$ and $e \gg f$, the $V$-shape parameter is close to 1 and the eclipse is $V$-shaped. One of the advantages of our modification is that the $V$-fit results in a better estimate of the transit depth. In addition, we use the $V$-shape parameter as a selection criterion to reject grazing eclipsing binary systems that have generally very large $V$ values (see next section).

We search for transit periods in the range between 0.5 and 12 days by using 100001 trial periods equally distributed in $1 / P$. To speed-up the calculation time, the folded light curves are re sampled to 200 bins. The fractional transit duration was tested between 0.006 and 0.1 phase units. For each input light curve, we detect the five best-fitting periods with the BLS algorithm and then perform the trapezoidal re fit for each of them. We then select the period that has the lowest $\chi_{\text {d.o.f. }}^{2}$ of the improved $V$-fit. Figure 7 shows the difference between the reduced $\chi_{\text {d.o.f. }}^{2}$ of the trapezoid-fit and the box-fit as a function of the $V$-shape parameter. The trapezoid-fit shows a significant improvement over the box-fit especially for high $V$ values.

\subsection{Selection criteria}

Due to the large number of light curves in the WTS, it is necessary to set up a number of selection criteria to automatize the selection of candidates and efficiently reduce the number of false positives in the survey. As an initial cut, we removed all objects with magnitudes $J>18$. Objects below $J$-band $=16$ are already difficult to follow-up; nevertheless, we decided to extend the magnitude cut $(J$-band $=17)$ used in Kovács et al. (2013) to make use of the improvement achieved by DI light curves for faint objects.

In addition, we reject objects for which the detection algorithm found a period that is close to alias periods introduced by the window function of the observing strategy. In particular, we exclude objects with periods in the ranges $0.485-0.515$, $0.985-1.015,1.985-2.015$ and $2.985-3.015$ days. As an example, Fig. 3 shows the high number of detections found around the one day alias period. For the sub-field $19 \mathrm{~g} 1$, we additionally exclude a narrow period range $1.350-1.352$ days due to a very high

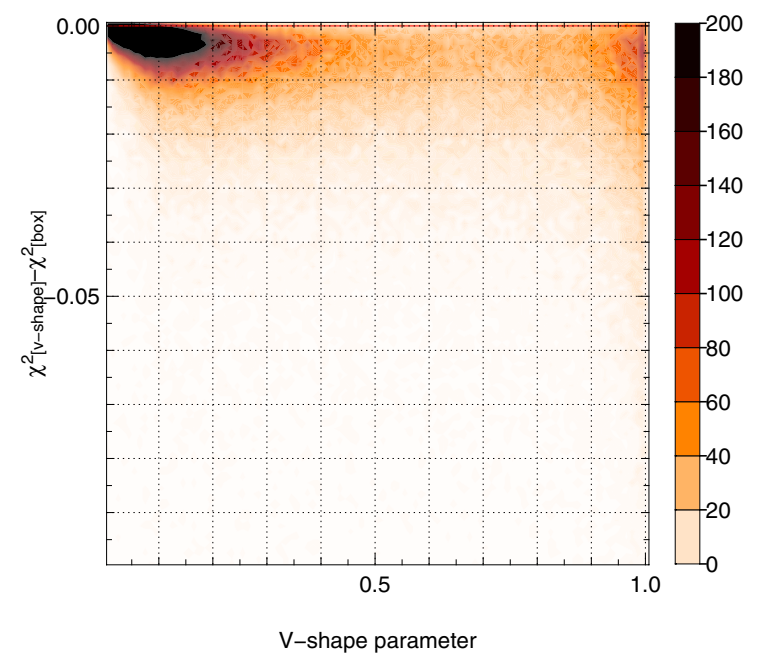

Fig. 7. $\chi_{\text {d.o.f. }}^{2}$ comparison between trapezoid-fit and box-fit for transit detections. Since the box-fit is actually included in the trapezoid-fit (i.e., $V=0$ ), positives values are not expected in this plot. On the other hand, a significant improvement in the trapezoid-fit is achieved specially for higher values of $V$.

number of false positives in this range. Based on our experience of previous works, we introduce six more selection criteria:

1. $S / N$ : one of the most important criteria is the signal-to-noiseratio $(\mathrm{S} / \mathrm{N})$ of the eclipse measured from the light curves. In the past, many authors have used different ways to calculate the $\mathrm{S} / \mathrm{N}$ and many different ways of utilizing it as a selection criterion. For instance, Burke et al. (2006) include the signalto-white-noise in their selection criteria to set the threshold to $S / N \geq 10$. Hartman et al. (2009) propose the same limit of $S / N \geq 10$, but the threshold corresponds to the pink noise (Pont et al. 2006) in their case. Kovács et al. (2013) use the red noise to fix the detection limit; they suggest a signal-tored noise of $S_{\text {red }} \geq 6$. Our $\mathrm{S} / \mathrm{N}$ selection criterion accounts only for white noise.

2. $S / N-S / N_{\text {rem }}$ : a large fraction of false positive detections are variable stars. To eliminate them, we use a new detection criterion, labeled $\mathrm{S} / \mathrm{N}-\mathrm{S} / \mathrm{N}_{\text {rem }}$, which is the difference of the $\mathrm{S} / \mathrm{N}$ found in the BLS analysis and the $\mathrm{S} / \mathrm{N}_{\text {rem }}$ found in a second pass of the algorithm after masking all points during the eclipse that has been detected in the first interaction. For a planet candidate, $\mathrm{S} / \mathrm{N}-\mathrm{S} / \mathrm{N}_{\text {rem }}$ is very high since the variability is confined to the transit phase. For variable stars, such as eclipsing binaries or sinusoidal variables, there is still variability left, which results in a low value of $S / N-S / N_{\text {rem }}$. Note that this criterion eliminates the detection of systems with more than one transiting planet. However, we decided to only search for systems with a single transiting planet, since the WTS survey is only sensitive to periods smaller than ten days.

3. Number of transit points: many light curves result in a high $\mathrm{S} / \mathrm{N}$ detection but only very few points belong to the transit. We therefore require a minimum number of transit points in our candidate selection process. Due to the scheduling of the WTS, we do not require a minimum number of individual transits as an additional criterion, since a small minimum number of transit points guarantees implicitly two transits or more.

4. $V_{\text {shape }}$ : one selection criterion that has not been used in previous studies is the $V$-shape parameter, which was defined in the previous section. The criterion acts as a filter to eliminate 
false positives generated by eclipsing binary systems. An eclipsing binary would be characterized by a very V-shaped eclipse, which has a high $V$ value.

5. Depth: some detections show a very deep transit signal. A typical brightness dimming that corresponds to a Sun-like star and a Jupiter-like planet is about $1 \%$. Transit signals that are much deeper are more likely to be eclipsing binary stars. Using a cut on the maximum allowed transit depth we reduce the number of false positive detections. For Jupiter-sized planets around M-dwarfs, the transit depth can be even higher than $10 \%$. We therefore optimize the detection criteria for M-stars independently (see below).

6. Transit duration: we impose a limit on the fractional transit duration to exclude candidates that show long eclipses, which are not physically realistic.

\subsection{Optimization of the selection criteria}

We optimize our selection criteria with Monte-Carlo simulations, where we inject transit signals in the real light curves using stellar parameter distributions (radius and mass) from the Besançon model of the galaxy (Robin et al. 2003) and limb-darkening coefficients from Claret \& Bloemen (2011). For each light curve of the WTS, we pick a random star from the Besançon model that has a similar magnitude $\left(\Delta_{\text {mag }} \leq 0.05\right)$ and draw a random period in the range from 0.5 to 12 days. Details about the transit injection procedure can be found in Koppenhoefer et al. (2009).

We split the light curves in two data sets, one for F-, G- and $\mathrm{K}$-stars and one for M-stars. The optimization of the selection criteria was done separately, since we expect some parameters to differ between both data sets. For instance, the transit depth is generally larger for planets that orbit M-dwarfs, and the fractional transit duration is smaller. In addition, the particular analysis of the M-dwarf sample allowed us to derive an upper limit on the occurrence rate of Jupiter-sized planets around low-mass stars (see Sect. 6).

The M-dwarf selection is based on color cuts in seven Sloan Digital Sky Survey data release 7 (SDSS 7th release, Adelman-McCarthy \& et al. 2009) and WFCAM bands: $g-r \geq$ $1.6, r-i \geq 0.9, i-z \geq 0.5, J-H \geq 0.45$ and $H-K \geq 0.17$. These cuts have been derived to include the majority of M-dwarfs selected by Kovács et al. (2013). Based on these cuts, we find $10375 \mathrm{M}$-stars brighter than $18 \mathrm{mag}$ in $J$-band. The number of objects with magnitudes brighter than $J=17$ is 4073 , which is slightly less but still in reasonable agreement with the number of M-dwarfs selected in Kovács et al. (2013), who found 4600 objects using an SED-fitting approach. In the following sections, we report the optimized selection criteria and the detection efficiency for both data sets and present and discuss the selected candidates.

\section{Candidates detected around F-, G-, and K-stars}

For the simulated light curves, we required the detected period to be within $1 \%$ of the simulated period, also allowing a value of half or double of this period. On a computer cluster, we ran 100 simulations in total. In each run, we considered each light curve once for a transit injection, resulting in about 1200000 simulated light curves. To save computation time, we simulated only those cases, in which the randomly drawn inclination vector results in a visible transit signal. After running the simulations, we optimized the selection criteria presented above for the DI and AP light curves of all F-, G- and K-stars. We allowed up to
Table 1. Objects removed by the optimized selection criteria from an original sample of 464873 DI light curves.

\begin{tabular}{lccc}
\hline \hline Criterion & Remaining objects & Removed objects & $\%$ \\
\hline$J \leq 18$ & 102428 & 362445 & 76.26 \\
Removed alias period & 72012 & 30416 & 29.69 \\
$S / N>18$ & 7080 & 64932 & 90.17 \\
$S / N-S / N_{\text {rem }}>8$ & 3391 & 3689 & 52.10 \\
Transit points $>24$ & 506 & 2285 & 85.08 \\
$V_{\text {shape }}<0.6$ & 288 & 218 & 43.08 \\
Depth $\leq 4 \%$ & 100 & 188 & 65.27 \\
Transit duration $\leq 0.5$ & 100 & 0 & 0.00 \\
\hline
\end{tabular}

Table 2. Objects removed by the optimized selection criteria from an original sample of 428928 AP light curves.

\begin{tabular}{lccc}
\hline \hline Criterion & Remaining objects & Removed objects & $\%$ \\
\hline$J \leq 18$ & 102428 & 326500 & 74.32 \\
Removed alias period & 73201 & 29227 & 28.53 \\
$S / N>11$ & 5778 & 67423 & 92.11 \\
$S / N-S / N_{\text {rem }}>6$ & 1760 & 4018 & 69.54 \\
Transit points $>18$ & 563 & 1197 & 68.01 \\
$V_{\text {shape }}<0.7$ & 360 & 203 & 36.06 \\
Depth $\leq 3 \%$ & 100 & 260 & 72.22 \\
Transit duration $\leq 0.5$ & 100 & 0 & 0.00 \\
\hline
\end{tabular}

100 detections on the unmodified light curves on each data set. This number is strategically selected, since it is small enough to allow a visual inspection of each detected object, while significantly larger than the expected number of planet detections.

Tables 1 and 2 list the optimized selection criteria for the DI and AP light curves for F-, G- and K-stars and provide the number of objects that remain after each of the selection criteria is applied. In this case, the fractional transit duration turned out to be a useless criterion to detect candidates around these stars. These selection criteria allow us to recover $10 / 26 \%$ of the signals injected into the AP/DI light curves with $S / N \sim 11 / 18$ (our minimum required $\mathrm{S} / \mathrm{N}$ ) and up to $80 / 80 \%$ with $S / N \geq 30 / 40$, respectively. The resulting total efficiencies are discussed in Sect. 5.4. Before applying the magnitude limit, we note that the number of light curves in the DI and AP data sets differ at the $10 \%$ level. This is because the object detection in the DI analysis was going slightly deeper than in the AP analysis.

To test whether the selection criteria differ from one to another detector, we initially optimize them for each of the sub fields independently but found almost identical values. We therefore decided to use one single set of selection criteria for F-, Gand K-stars in the whole $19 \mathrm{~h}$ field.

We visually inspect the 200 detections that pass the optimized selection criteria in the AP and DI data sets to remove candidates that are clear eclipsing binaries with two eclipses of different depth. We also reject objects that show significant out of eclipse variations, very asymmetric eclipse shapes, and candidates which are too noisy to be further analyzed. Our final list of candidates includes eleven objects, of which seven were detected in the AP-light curves and six are from the DI light curves. Two objects were detected in both the DI and AP light curves. One of this detections is WTS-2b that has recently been confirmed as a planet by the Rocky Planets Around Cool Stars ${ }^{4}$ (RoPACS)

\footnotetext{
4 RoPACS is a Marie Curie initial training network. The research of the RoPACS community is based on the data obtained by the WTS.
} 
Table 3. List of new candidates around F-G-K stars detected in this work.

\begin{tabular}{|c|c|c|c|c|c|c|c|c|c|c|c|c|c|}
\hline Object & Data-set & $\alpha$ & $\delta$ & $u$ & $g$ & $r$ & $i$ & $z$ & $Z$ & $Y$ & $J$ & $H$ & $K$ \\
\hline 19b1-02162 & AP/DI & 293.0112 & 36.4848 & 19.00 & 17.73 & 17.13 & 16.89 & 16.76 & 16.37 & 16.22 & 15.93 & 15.49 & 15.37 \\
\hline 19f3-06991 & $\mathrm{AP}$ & 293.4682 & 36.4995 & 15.97 & 14.66 & 14.26 & 14.13 & 14.07 & 13.62 & 13.56 & 13.34 & 13.07 & 13.02 \\
\hline 19b3-09004 & DI & 293.5208 & 36.8839 & 17.97 & 16.60 & 16.03 & 15.80 & 15.67 & 15.25 & 15.17 & 14.85 & 14.45 & 14.39 \\
\hline $19 \mathrm{~g} 1-11212$ & AP & 293.6753 & 36.1420 & 16.57 & 15.40 & 14.93 & 14.81 & 14.74 & 14.32 & 14.25 & 14.01 & 13.73 & 13.65 \\
\hline $19 \mathrm{c} 4-02952$ & DI & 293.8666 & 36.7571 & 18.53 & 17.38 & 16.97 & 16.79 & 16.70 & 16.23 & 16.11 & 15.83 & 15.51 & 15.46 \\
\hline $19 \mathrm{~h} 1$ & AP & 294.1531 & 36.0794 & 19.55 & 17.18 & 16.19 & 15.84 & 15.60 & 15.28 & 15.03 & 14.60 & 14.09 & 13.91 \\
\hline $19 \mathrm{~b} 3$ & $\mathrm{AP}$ & 293.4401 & 36.7404 & 20.51 & 18.67 & 18.08 & 17.81 & 17.65 & 17.24 & 17.12 & 16.78 & 16.40 & 16.33 \\
\hline $19 \mathrm{e} 1$ & DI & 292.6870 & 36.2186 & 18.04 & 17.09 & 16.54 & 16.29 & 16.20 & 15.84 & 15.73 & 15.42 & 15.08 & 15.01 \\
\hline $19 b 4$ & AP & 292.9365 & 36.7902 & 17.04 & 15.70 & 15.18 & 14.95 & 14.86 & 14.38 & 14.28 & 13.96 & 13.54 & 13.47 \\
\hline $19 \mathrm{~b} 2-01819$ & DI & 293.5220 & 36.4675 & 18.27 & 16.95 & 16.48 & 16.32 & 16.25 & 15.86 & 15.75 & 15.46 & 15.13 & 15.07 \\
\hline
\end{tabular}

Notes. The second column shows the light curve data set in which the candidates have been detected. The coordinates (J2000.0) are listed in Cols. 3 and 4. The remaining columns provide broadband photometric measurements of our candidates in ten different filters. The $u, g, r, i, z$ $A B$-magnitudes were obtained from the SDSS and the $Z, Y, J, H, K$ magnitudes are WFCAM measurements in the Vega-system.

community (Birkby et al. 2013a,b). The other planet that has been found in the WTS (Cappetta et al. 2012) is WTS-1b, which not detected by our selection criteria due to a very low $\mathrm{S} / \mathrm{N}$ value.

In the following, we present a detailed analysis of the ten remaining candidates and include a characterization of the host stars, a light curve fit with an analytic transit model, and a test for double-eclipse binary scenarios. The analysis provides important physical parameters of the host stars and companions, which are used to asses the quality of the candidates. Figure 16 shows the folded light curves of our candidates.

\subsection{Characterization of the host star}

The broadband photometric measurements of the host stars of the candidates are listed in Table 3. The WFCAM provides photometry in five bands $(Z, Y, J, H, K)$. Additional measurements in five optical bands $(u, g, r, i, z)$ were obtained from the database of the SDSS). The table also shows the data set, in which the candidates were detected (AP or DI). The candidate 19b1-02162 was found in both AP and DI data sets; in this case, we use the AP light curve in the following, since it presents a lower scatter.

The characterization of the host star is essential for inferring physical properties of the candidates, such as planetary radius and orbit inclination. The Virtual Observatory SED Analyzer ${ }^{5}$ (VOSA, Bayo et al. 2008) is an on-line tool designed to automatically perform several tasks, such as the determination of stellar parameters by analyzing the SED. This analysis was carried out in our candidates using the photometry reported in Table 3. The VOSA works with input parameters that can be submitted as ASCII files. They must include a reference name of the source, coordinates, visual extinction $A_{\mathrm{v}}$, filter names, observed fluxes, and the corresponding errors. Although the VOSA enables us to select among six different fitting models, only two are appropriate for our purpose. For the F-, G- and $\mathrm{K}$-stars, we adopt the Kurucz ATLAS9 templates described in Castelli et al. (1997), which provide better results for a wider temperature range than the NextGen model (Baraffe et al. 1998). The program offers the option of restricting free parameters ( $T_{\text {eff }}, \log g$ and $\left.[\mathrm{Fe} / \mathrm{H}]\right)$ to speed up the fitting process. We confine the limits to $T_{\text {eff }}=3500-10000 \mathrm{~K},[\mathrm{Fe} / \mathrm{H}]=0.0$ and $\log g=3.5-5.0$. Note that the selected values of $T_{\text {eff }}$ and $\log g$ are compatible with main-sequence stars with spectral types between $\mathrm{A}$ and $\mathrm{M}$. The program compares the broadband

\footnotetext{
5 http://svo2.cab.inta-csic.es/theory/vosa/
}

photometric measurements to theoretical synthetic spectra to find the best-fitting SED. The VOSA tests a large range of stellar models within the given parameter limits. The SED-fit is also sensitive to the extinction $A_{\mathrm{v}}$, which is used as an additional free parameter. The extinction and the corresponding SED model are obtained by testing 100 different $A_{\mathrm{v}}$ values, which are distributed in a range from 0.01 to $1 \mathrm{mag}$. We select the values that result in the lowest $\chi^{2}$ within the valid extinction range from 0.01 up to the maximum allowed extinction that is set by the total Galactic extinction. This upper limit is obtained from the Galactic extinction calculator of the NASA/IPAC extragalactic database ${ }^{6}$ (see Fig. 8). In some cases, the absolute minimum corresponds to an extinction that is higher than the upper limit. For these cases, we select the best solution that are within the allowed range. In Tables 4 and 10, we mark these particular cases with an asterisk. The resulting best-fitting model provides an estimate of the $T_{\text {eff }}$ of the host stars, which are also summarized in Tables 4 and 10 . The results show that the $T_{\text {eff }}$ of the parent stars are in the range of 4750-6500 K, which corresponds to spectral types between $\mathrm{K} 3$ and F5. According to the $T_{\text {eff }}$ found in the fit, we derive stellar radii and masses and calculate the surface gravity $\log g$ using 1-5 Gyr isochrones for solar metallicity obtained from the Dartmouth stellar evolution database (Dotter et al. 2008). These values are reported in Tables 4 and 10 as $R 1_{\star}, M_{\star}$, and $\log g_{1}$. The error ranges of the stellar radii is determined by assuming a precision of $250 \mathrm{~K}$, which is the step size of the grid used in the VOSA fit. Figure 9 shows an example of the VOSA fit of our best candidate 19b1-02162.

\subsection{Secondary eclipse fit}

For each candidate, we tested the possibility that we actually detected an eclipsing binary system with similar eclipse depths, where the primary and secondary eclipse have been folded together at half the binary period. To carry out this test, we fold the light curve of each candidate with double the detected period, and fit a primary and secondary eclipse which are offset by 0.5 phase units assuming a circular orbit. Under this assumption, our candidate sample may be contaminated with eclipsing binaries in high eccentric orbits. However, Devor (2005) shows that only $\sim 10 \%$ of the binaries studied there with periods shorter than 12 days have eccentricities higher than 0.1 . Therefore, the possible contamination is low to start with. Moreover, any

6 http://ned.ipac.caltech.edu/forms/calculator.html 


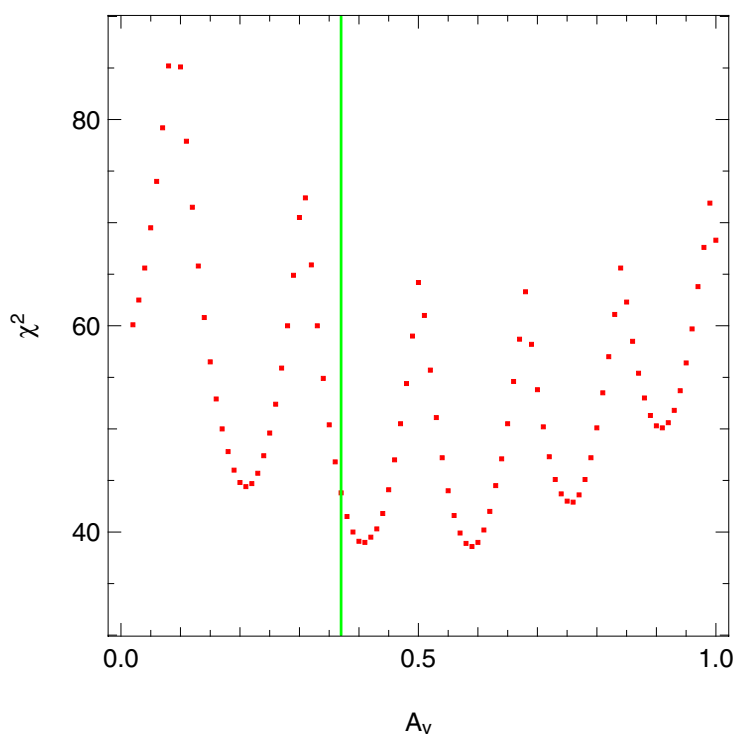

Fig. 8. $\chi^{2}$ as a function of the input visual extinction value used in the SED fit of our planet candidate 19b1-02162. Although the value of $A_{\mathrm{v}} \sim 0.6$ mag results in the lowest $\chi^{2}$, we use the value of $A_{\mathrm{v}}=$ $0.21 \mathrm{mag}$, which is based on the upper limit extinction adopted from the NASA/IPAC Extragalactic Database, since an extinction of $A_{\mathrm{v}}=$ 0.6 mag would be physically non-realistic. The upper limit mentioned above is shown with the green solid line. The periodic distribution of the $\chi^{2}$ is due to the variation of six different stellar spectral-types.

candidate with a clear deeper secondary eclipse would be rejected during our visual inspection. Both the primary and secondary eclipses are first fitted with a box and subsequently refitted with a symmetrical trapezoid, as described in Sect. 4. A significant difference between the depths of the primary and secondary eclipse indicates that the candidate could be an eclipsing binary rather than a star with a planet. Also a comparison of the $\chi_{\text {d.o.f. }}^{\prime 2}$ of the binary fit to the $\chi_{\text {d.o.f. }}^{2}$ of the fit with the planet period can indicate that the candidate is actually a binary with similar eclipse depths. We would like to point out that the decision of presenting either the planet or binary periods includes a visual examination of the folded light curves (Figs. 16 and 17). This inspection showed that $\chi_{\text {d.o.f. }}^{2}$ and eclipse depth differences cannot be used blindly for the discrimination, since they closely depend on the number of points during the eclipses and box-fitting parameters. Note that the trapezium fit is only a crude model of a transit light curve, and we found that the depth estimated by our algorithm did not reflect the true depth as one sees in the folded light curves in some cases. In summary, the discrimination between both scenarios based on the $\chi_{\text {d.o.f. }}^{2}$ and eclipse depth values is only used as a hint for selecting either the planet or binary period rather than a decisive proof of the nature of the candidate. The final decision to classify our candidates was done case-bycase and primarily based on the best-fitting radius as found in the analytic transit fit (see Sect. 5.3). Table 5 summarizes the results of the secondary eclipse fit analysis.

\subsection{Transit fit}

We carried out an improved fit to the $J$-band light curves of the candidates using analytic transit models proposed by Mandel \& Agol (2002). For two candidates (19b1-02162 and 19b2-01819), we additionally used an $i^{\prime}$-band light curve, covering one full eclipse, which was obtained in a photometric follow-up campaign at the INT in La Palma. In these cases, we performed

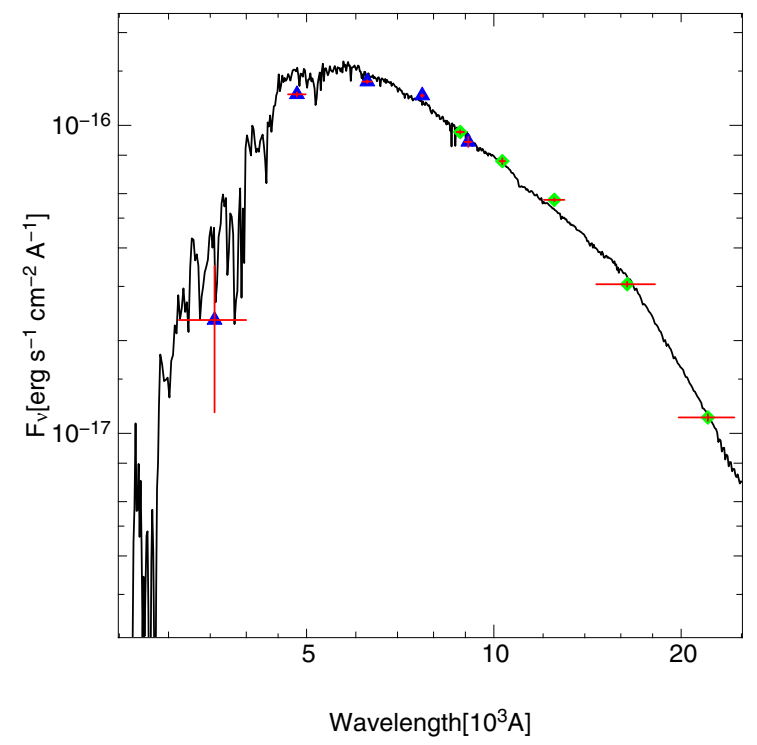

Fig. 9. Best Kurucz ATLAS9 model derived with the VOSA (black line) for the SED of 19b1-02162. The effective temperature of the best-fitting model is $T_{\text {eff }}=5500 \mathrm{~K}$ for an extinction of $A_{\mathrm{v}}=0.21$. Blue triangles represent the SDSS photometry, while green diamonds correspond to the WFCAM photometry. Vertical and horizontal errors bars are the flux uncertainties and the equivalent width of each pass band.

a simultaneous fit to both light curves. The transit light curve model depends on quadratic limb-darkening coefficients, which were deduced from the linear interpolations in $T_{\text {eff }}$ and $\log g$ of the values listed in Claret \& Bloemen (2011). We used the $T_{\text {eff }}$ of host stars that were previously obtained by the SED analysis (see Sect. 5.1) and the corresponding $\log g$ values from the 1-5 Gyr isochrones, assuming a solar metalicity $[\mathrm{Fe} / \mathrm{H}]=0.0$ and a micro turbulence of $2 \mathrm{~km} \mathrm{~s}^{-1}$. We utilized the values derived from ATLAS atmospheric models using the flux conservation method (FCM). Alternatively, the values can be derived using the least-squares method (LSM). However, a test of the analytic transit fit was carried out by using the values from the two different models. The results showed the same goodness of the fit for both methods, so we have chosen the FCM over the LSM model without any specific preference. Using the WTS $J$-band light curve, we fitted the mean stellar density $\rho_{\star} \sim M_{\star} / R_{\star}^{3}$ in solar units, the radius ratio $R_{\text {planet }} / R_{\star}$, the impact parameter $\beta_{\text {impact }}$ in units of $R_{\star}$, the orbital period $P$, and epoch of the central transit $t_{0}$. The iterative fitting process required starting values for a series of input parameters, such as period, epoch of transit, planet radius, and parameters related to the stellar companion, such as mass and radius. The period, epoch of transit, and planet radius were obtained directly from the results provided by our transit detection algorithm, while the stellar parameters $\left(R 1_{\star}\right.$ and $M_{\star}$ ) were estimated by using the previously fitted $T_{\text {eff }}$ from the 1-5 Gyr model isochrones for solar metalicity (Dotter et al. 2008). From the best-fit of the analytic transit model, we were able to calculate the intrinsic physical parameters of the candidates and host stars, such as $R_{\text {planet }}$ and $R 2_{\star}$.

The fitting procedure also enabled us to derive an error estimation of the fitted parameters. The errors were calculated using a multi-dimensional grid in which we searched for extreme points with $\Delta \chi^{2}=1$. This method corresponds to a variation of each single parameter, while minimizing over the others. The results of the transit fit are listed in Tables 4 and 6 . Figures 10 and 11 show the best-fitting model of our best candidate $19 \mathrm{~b}-1-02162$ in the $J$ and $i^{\prime}$-bands, respectively. 
Table 4. Characterization of host stars.

\begin{tabular}{lccccccccc}
\hline \hline Object & $T_{\text {eff }}(\mathrm{K})$ & Spectral type & $\log g_{1}$ & $\log g_{2}$ & $A_{\mathrm{v}}$ & Distance $(\mathrm{pc})$ & $R 1_{\star}\left(R_{\odot}\right)$ & $R 2_{\star}\left(R_{\odot}\right)$ & $M_{\star}\left(M_{\odot}\right)$ \\
\hline $19 \mathrm{~b} 1-02162$ & 5500 & $\mathrm{G} 8$ & 4.56 & 4.32 & $0.21^{*}$ & 2188 & $0.85_{-0.05}^{+0.07}$ & $1.12_{-0.12}^{+0.07}$ & $0.95_{-0.06}^{+0.07}$ \\
$19 \mathrm{f} 3-06991$ & 6500 & $\mathrm{~F} 5$ & 4.31 & 4.05 & 0.35 & 1127 & $1.23_{-0.10}^{+0.20}$ & $1.74_{-0.16}^{+0.38}$ & $1.25_{-0.08}^{+0.13}$ \\
$19 \mathrm{~b} 3-09004$ & 5750 & $\mathrm{G} 5$ & 4.52 & 4.27 & 0.28 & 1472 & $0.92_{-0.07}^{+0.08}$ & $1.22_{-0.04}^{+0.03}$ & $1.02_{-0.07}^{+0.07}$ \\
$19 \mathrm{~g} 1-11212$ & 6250 & $\mathrm{~F} 7$ & 4.41 & 4.00 & $0.22^{*}$ & 1330 & $1.13_{-0.12}^{+0.20}$ & $1.78_{-0.18}^{+0.27}$ & $1.17_{-0.08}^{+0.09}$ \\
$19 \mathrm{c} 4-02952$ & 6250 & $\mathrm{~F} 7$ & 4.41 & 4.01 & $0.44^{*}$ & 3119 & $1.13_{-0.12}^{+0.20}$ & $1.77_{-0.07}^{+0.11}$ & $1.17_{-0.08}^{+0.09}$ \\
$19 \mathrm{~h} 1-00325$ & 4750 & $\mathrm{~K} 3$ & 4.57 & 4.43 & 0.16 & 773 & $0.71_{-0.04}^{+0.03}$ & $0.89_{-0.05}^{+0.08}$ & $0.78_{-0.05}^{+0.04}$ \\
$19 \mathrm{~b} 3-05398$ & 6000 & $\mathrm{G} 0$ & 4.47 & 4.19 & 0.45 & 4345 & $1.00_{-0.08}^{+0.13}$ & $1.39_{-0.07}^{+0.08}$ & $1.09_{-0.07}^{+0.11}$ \\
$19 \mathrm{e} 1-05755$ & 6000 & $\mathrm{G} 0$ & 4.47 & 4.12 & 0.38 & 2208 & $1.00_{-0.08}^{+0.13}$ & $1.51_{-0.14}^{+0.06}$ & $1.09_{-0.07}^{+0.11}$ \\
$19 \mathrm{~b} 4-04138$ & 5750 & $\mathrm{G} 5$ & 4.52 & 3.96 & 0.45 & 506 & $0.92_{-0.07}^{+0.08}$ & $1.74_{-0.05}^{+0.03}$ & $1.02_{-0.07}^{+0.07}$ \\
$19 \mathrm{~b} 2-01819$ & 6250 & $\mathrm{~F} 7$ & 4.41 & 3.93 & 0.38 & 2559 & $1.13_{-0.12}^{+0.20}$ & $1.94_{-0.05}^{+0.04}$ & $1.17_{-0.08}^{+0.09}$ \\
\hline
\end{tabular}

Notes. The $T_{\text {eff }}$ is derived from SED-fit. We use 1-5 Gyr isochrones obtained from the Dartmouth stellar evolution database (Dotter et al. 2008) to estimate $R 1_{\star}, \log g_{1}$ and $M_{\star}$. The extinction values $\left(A_{v}\right)$ found in the SED-fit are reported in Col. 6 . In the three cases marked with an asterisk, the best-fitting extinction is higher than the total extragalactic extinction and we report the extinction that corresponds to the minimum $\chi^{2}$ within the allowed extinction range. The stellar radii $R 2_{\star}$ correspond to the best-fitting analytic transit model (see Sect. 5.3). The values of log $g_{2}$ reported in Col. 5 are estimated from the stellar radii $R 2_{\star}$, which tend to be higher than $R 1_{\star}$, resulting in lower $\log g_{2}$. The distances reported in Col. 7 are estimated utilizing the extinction values found in the VOSA analysis, the $i$-band magnitudes reported in Table 3 , and the absolute magnitudes $M_{\mathrm{i}}$, which are obtained from the isochrones.

Table 5. Comparison between the planet and binary scenarios.

\begin{tabular}{lcccccccc}
\hline \hline Object & $V$ & $\mathrm{dp}(\%)$ & $\chi_{\text {d.o.f. }}^{2}$ & $\chi_{\text {d.o.f. }}^{\prime 2}$ & $\mathrm{dp}_{1}^{\prime}(\%)$ & $\mathrm{dp}_{2}^{\prime}(\%)$ & $V_{1}^{\prime}$ & $V_{2}^{\prime}$ \\
\hline $19 \mathrm{~b} 1-02162$ & 0.25 & 2.05 & 1.3792 & 1.3438 & 2.54 & 1.37 & 0.25 & 0.00 \\
$19 \mathrm{f} 3-06991$ & 0.56 & 0.81 & 1.0087 & 0.9545 & 1.08 & 0.47 & 0.58 & 0.33 \\
$19 \mathrm{~b} 3-09004$ & 0.31 & 3.07 & 4.3247 & 4.2817 & 3.44 & 2.87 & 0.54 & 0.01 \\
$19 \mathrm{~g} 1-11212$ & 0.37 & 1.49 & 1.4751 & 1.4301 & 2.29 & 1.34 & 0.45 & 0.81 \\
$19 \mathrm{c} 4-02952$ & 0.57 & 4.13 & 3.3622 & 3.3617 & 3.73 & 3.86 & 0.00 & 0.53 \\
$19 \mathrm{~h} 1-00325$ & 0.43 & 3.11 & 4.0514 & 4.0172 & 2.92 & 3.05 & 0.16 & 0.38 \\
$19 \mathrm{~b} 3-05398$ & 0.29 & 2.66 & 0.9802 & 0.9739 & 2.91 & 2.55 & 0.48 & 0.36 \\
$19 \mathrm{e} 1-05755$ & 0.29 & 1.76 & 1.7486 & 1.7211 & 2.54 & 1.66 & 0.80 & 0.54 \\
$19 \mathrm{~b} 4-04138$ & 0.64 & 2.53 & 1.7270 & 1.7041 & 2.51 & 2.36 & 0.66 & 0.62 \\
$19 \mathrm{~b} 2-01819$ & 0.45 & 2.80 & 2.7123 & 2.6819 & 2.74 & 3.07 & 0.61 & 0.65 \\
\hline
\end{tabular}

Notes. Comparison of the eclipse shapes, eclipse depths, and $\chi_{\text {d.o.f. }}^{2}$ values of the planet scenario and binary scenario (prime values on the right side of the table).

\subsection{Discussion of the candidates}

Table 6 provides a list of our candidates sorted, according to their best-fitting radius. All candidates except for the first two have very large best-fitting radii, larger than all transiting planets published so far. We therefore conclude that they are systems with a transiting brown dwarf or a low-mass stellar companion.

The first two candidates have best-fitting radii of $1.61 R_{\text {Jup }}$ and $1.65 R_{\text {Jup }}$; however, the secondary eclipse fit results in a slightly better $\chi_{\text {d.o.f. }}^{2}$ for the binary scenario, and the primary and secondary eclipses show different depths, which are hints to select the binary period instead of the planet scenario. By looking at the folded light curves (Fig. 16), the second candidate (19f3-06991) is a clear case where the fit with the binary period reveals two well sampled eclipses with different depths. The first candidate (19b1-02162) is not as clear. Although the binary period fit shows two different eclipses with depths of 2.5 and $1.4 \%$, the single eclipse observed in the $i^{\prime}$-band coincides with the deeper eclipse, but has a depth of $1.8 \%$ (see Fig. 11), which is closer to the shallower eclipse. We therefore conclude that the correct period is unclear for this candidate, and we propose it as a target for high precision photometric follow-up. Figure 12 shows a $J$-band image of 19b1-02162.

To estimate the number of planets that we expect to find, we calculate the overall detection efficiency in our simulations, which are $\sim 1.7 \%$ and $\sim 2.4 \%$ for DI and AP light curves, respectively. Accounting for an average geometrical probability of $11.9 \%$ to see transits (as derived from our Monte-Carlo simulations) and using an occurance rate for short period Jupiter-sized planets of $0.5 \%$ (Gould et al. 2006; Howard et al. 2012), we estimate the number of planets that we expect to find in the whole sample of 102428 light curves to be 1.0 (DI) and 1.5 (AP). This is in very good agreement with the two planets that have been detected in the WTS so far (Cappetta et al. 2012; Birkby et al. 2013a,b).

\section{Candidates detected around M-stars}

\subsection{Selection criteria for $M$-stars}

We optimized the selection criteria for M-stars by injecting artificial transit signals into the DI and AP light curves of our 
Table 6. Results of the transit fit.

\begin{tabular}{lcccccccc}
\hline \hline Candidate & Period (days) & $t_{0}$ & $i\left(^{\circ}\right)$ & $R_{\text {planet }}\left(R_{\text {Jup }}\right)$ & $R_{\text {planet,min }}\left(R_{\text {Jup }}\right)$ & $R_{\text {planet,max }}\left(R_{\text {Jup }}\right)$ & $\chi_{\text {d.o.f. }}^{2}$ & Classification \\
\hline 19b1-02162 & 0.59862739 & 2454317.7883529 & 72.01 & 1.61 & 1.40 & 1.97 & 1.31 & $\mathrm{P}$ \\
$19 \mathrm{f} 3-06991$ & 0.71482077 & 2454318.3894489 & 66.54 & 1.65 & 1.43 & 3.26 & 1.00 & $\mathrm{~B}$ \\
$19 \mathrm{~b} 3-09004$ & 3.55921358 & 2454320.9406801 & 84.31 & 2.22 & 2.10 & 2.32 & 2.07 & $\mathrm{~B}$ \\
$19 \mathrm{~g} 1-11212$ & 2.77301797 & 2454318.9644598 & 80.23 & 2.29 & 3.44 & 1.94 & 1.21 & $\mathrm{~B}$ \\
$19 \mathrm{c} 4-02952$ & 3.42965118 & 2454319.6314035 & 82.25 & 3.54 & 3.36 & 3.81 & 1.84 & $\mathrm{~B}$ \\
$19 \mathrm{~h} 1-00325$ & 0.80767863 & 2454318.2698431 & 72.23 & 3.97 & 2.10 & 4.20 & 2.01 & $\mathrm{~B}$ \\
$19 \mathrm{~b} 3-05398$ & 0.73369311 & 2454317.9513207 & 67.12 & 4.20 & 3.53 & 4.99 & 1.18 & $\mathrm{~B}$ \\
$19 \mathrm{e} 1-05755$ & 0.77250704 & 2454318.1282676 & 64.34 & 5.30 & 3.00 & 13.37 & 1.21 & $\mathrm{~B}$ \\
$19 \mathrm{~b} 4-04138$ & 1.10663897 & 2454318.0883407 & 67.34 & 6.14 & 4.17 & 8.32 & 1.30 & $\mathrm{~B}$ \\
$19 \mathrm{~b} 2-01819$ & 0.82989549 & 2454318.2785263 & 53.23 & 15.59 & 15.43 & 15.98 & 1.27 & $\mathrm{~B}$ \\
\hline
\end{tabular}

Notes. Orbital and planetary parameters derived from the analytic transit model. Only one candidate, 19b-1-02162, is considered to be a planet candidate. All other candidates are too large and most likely transiting brown dwarfs or low-mass stars.

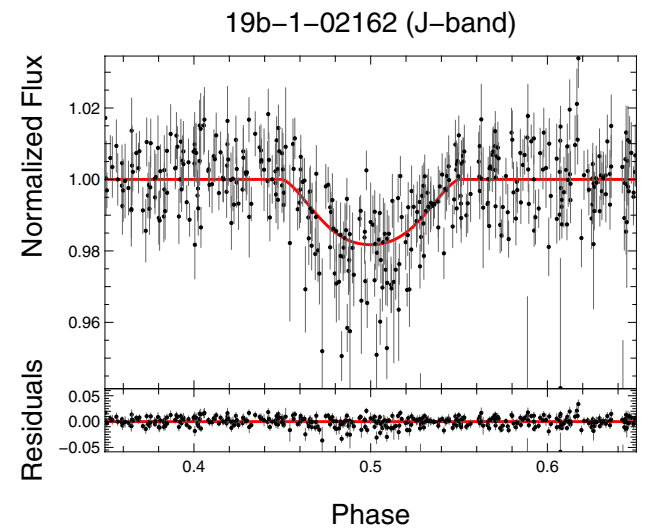

Fig. 10. Best-fitting model of 19b-1-02162 using the $J$-band light curve. The top frame shows the best-fit, whereas the bottom frame represents the residuals of the fit.

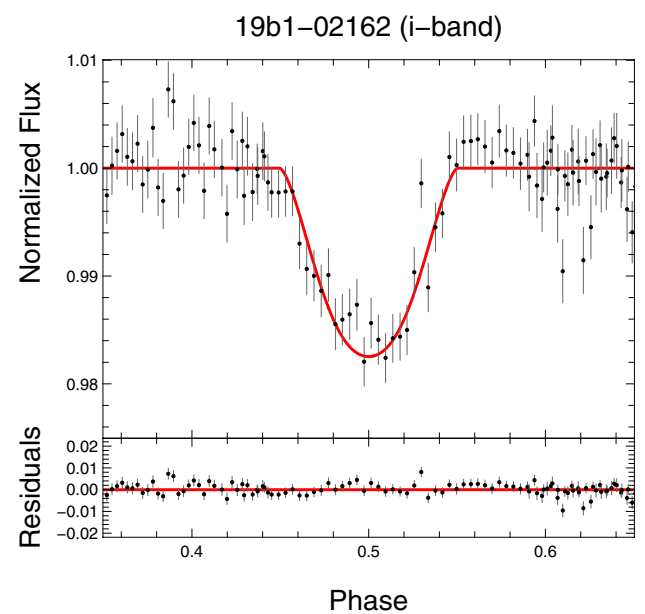

Fig. 11. Best-fit of 19b-1-02162 using the $i^{\prime}$-band light curve.

WTS M-star sample. The sample was selected using color cuts (see above). The simulated planet radius was always $1 R_{\text {Jup }}$, and we used a flat period distribution between 0.8 and 10 days. Using the criteria presented above, we optimized the selection of M-dwarf planet candidates for the DI and AP light curves, allowing up to 200 detections on the unmodified light curves. As for the F-, G- and K-stars, we require the detected period to differ by $1 \%$ from the simulated period, allowing also half or double of this value.

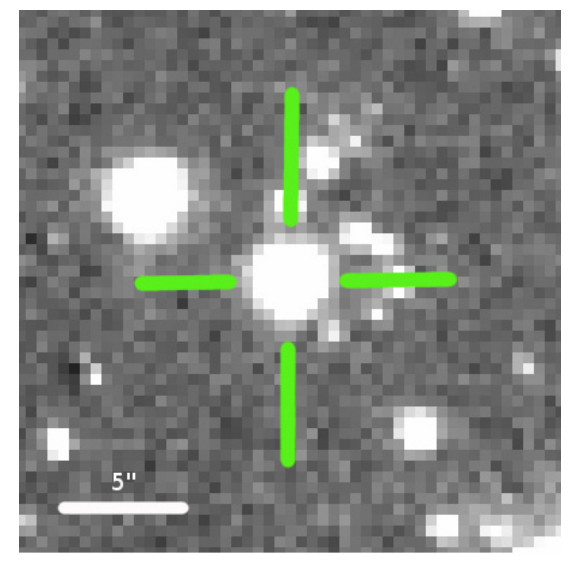

Fig. 12. Zoom-in showing the crowded neighborhood of the candidate 19b1-02162 in the sky. Our DI pipeline is optimized to deal with such cases.

Table 7. Optimized selection criteria for the DI M-star sample.

\begin{tabular}{lccc}
\hline \hline Criterion & Remaining objects & Removed objects & $\%$ \\
\hline$J \leq 18$ & 10375 & $\ldots$ & $\ldots$ \\
Removed alias period & 7913 & 2462 & 23.73 \\
$S / N>12$ & 1450 & 6463 & 81.68 \\
$S / N-S / N_{\text {rem }}>5$ & 536 & 914 & 63.03 \\
Transit points $>8$ & 164 & 372 & 69.40 \\
$V_{\text {shape }}<1.00$ & 164 & 0 & 0.00 \\
Depth $\leq 30 \%$ & 138 & 26 & 15.85 \\
Transit duration $\leq 0.06$ & 98 & 40 & 28.98 \\
\hline
\end{tabular}

Tables 7 and 8 list the optimized criteria for the DI and AP light curves. Unlike the case of F-, G- and K-stars, the fractional transit duration turned out to be a useful selection criterion. The $V_{\text {shape }}$ parameter turned out not to be important since transits of Jupiter-sized planets orbiting M-dwarfs can be very $V$-shaped.

Figure 14 shows the detection efficiency as a function of the apparent host star magnitude. Since the total number of M-stars is dominated by the faint end of the magnitude distribution, the overall efficiency of the DI light curves is slightly higher with $44.8 \%$ with respect to $43.8 \%$ for AP light curves. Figure 13 shows the efficiency of the DI light curves as a function of the number of detections on the unmodified light curves. Allowing 100 detections provides a high efficiency, while still being manageable to visually inspect. 
Table 8. Optimized selection criteria for the AP M-star sample.

\begin{tabular}{lccc}
\hline \hline Criterion & Remaining objects & Removed objects & $\%$ \\
\hline$J \leq 18$ & 10375 & $\ldots$ & $\ldots$ \\
Removed alias period & 8510 & 1865 & 17.98 \\
$S / N>6$ & 4411 & 4099 & 48.17 \\
$S / N-S / N_{\text {rem }}>2$ & 278 & 4133 & 93.70 \\
Transit points $>12$ & 168 & 110 & 39.57 \\
$V_{\text {shape }}<1.0$ & 168 & 0 & 0.00 \\
Depth $\leq 30 \%$ & 161 & 7 & 4.17 \\
Transit duration $\leq 0.08$ & 98 & 63 & 39.13 \\
\hline
\end{tabular}

After visual examination of the 200 automatically selected candidates from the AP and DI light curves, we identified eight possible candidates. All of them were found in both the AP and DI light curves. Table 9 lists the coordinates and broadband photometric data. As for the candidates found in the F-, G- and $\mathrm{K}$-star sample, we performed three different types of analyses to further asses the possibility of them being transiting planets. The result from the characterization of the host stars is shown in Table 10. For the M-dwarf analysis, we used the NextGen model atmosphere grid, which provides a wider range for low $T_{\text {eff }}$, which are more appropriated for M-dwarfs. In this case, we restrict the limits to $T_{\text {eff }}=1800-4500 \mathrm{~K},[\mathrm{Fe} / \mathrm{H}]=0.0$, and $\log g=4.5-5.5$. All eight stars are in the range of $3300 \mathrm{~K} \leq$ $T_{\text {eff }} \leq 3900 \mathrm{~K}$, corresponding to spectral types M5 to M0.

In the next step, we carried out a comparative analysis between the planet and binary scenarios, as described in Sect. 5.2. The results are shown in Table 11, which reveal different eclipse

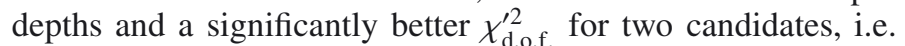
19a1-02980 and 19a1-10878.

Finally, we fit the $J$-band light curves of the eight candidates with an analytic transit model (see Sect. 5.3). For all faint candidates with $J \geq 17 \mathrm{mag}$, we used the DI light curve since the photometric precision is higher compared to the AP light curves. For the brighter candidates 19a1-02980 and 19a1-10878, we used the AP light curves. We determined the best-fitting period, epoch of transit, orbital inclination, and planet radius. The resulting values are listed in Table 12, where the smallest of our candidates has a radius of $2.53 R_{\text {Jup }}$, which exceeds the radius of any planet previously reported. We show the folded light curves of our candidates around M-dwarfs in Fig. 17.

Since none of our candidates has a best-fitting radius in the planetary regime, we conclude that they are all transiting brown dwarfs or low-mass stars, and we therefore confirm the hypothesis presented in Kovács et al. (2013) about the null detection of Jupiter-sized planets around M-dwarfs in the WTS. Following their approach, we derived a $95 \%$ confidence upper limit on the giant planet occurrence rate for M-dwarfs. Kovács et al. (2013) analyzed all sources with $J \leq 17$ mag and found an upper limit of 1.7-2.0\% for M0-M4 spectral types. In this work, we extended the search to all M-type stars with $J \leq 18$ mag. The extra magnitude bin increased the number of sources by a factor of 2.8 . In addition, we introduced an automatic selection procedure that reduces the number of candidates to be visually inspected to 100 for each set of light curves.

Assuming none of the candidates presented above are planets, we set an upper limit on the giant planet occurrence rate. Using Eq. (6) of Kovács et al. (2013), the overall detection efficiency of $44.8 \%$ for DI light curves, the average geometrical probability to see eclipses, and the total number of sources of 10375 , we find that the resulting upper limit is $1.1 \%$.

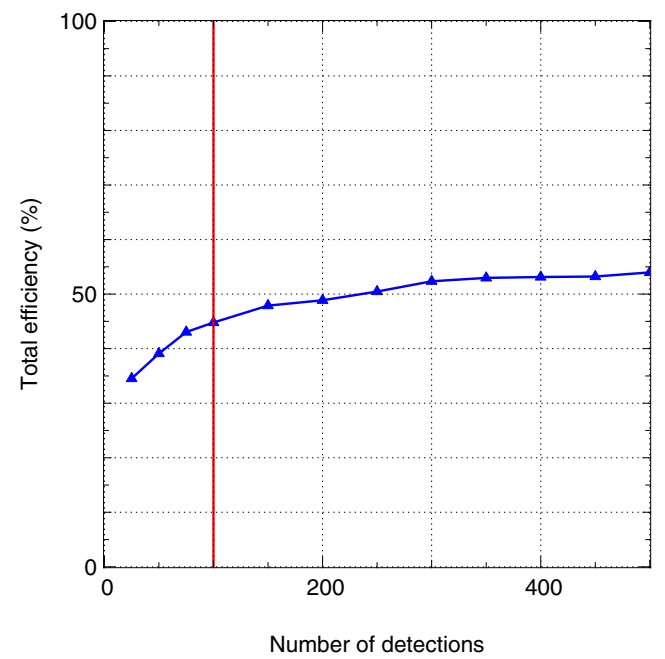

Fig. 13. Optimized total detection efficiency as a function of the number of detections on the unmodified DI light curves. The red line shows our limit of 100 detections.

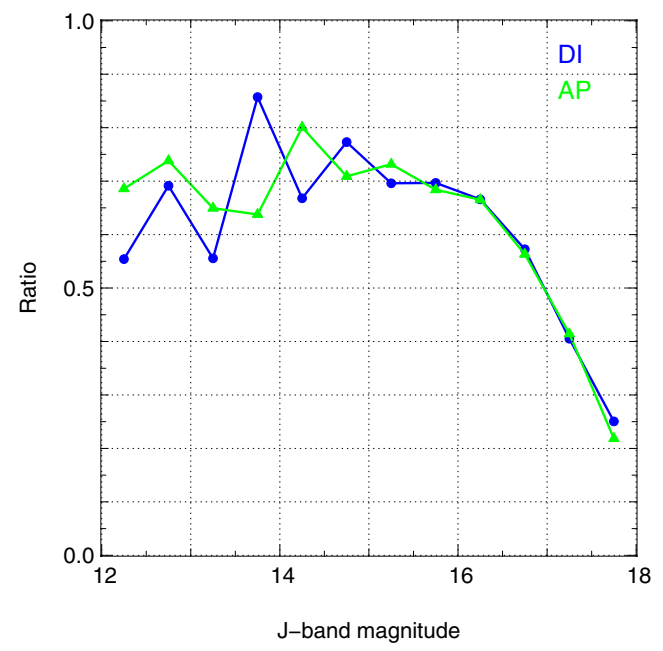

Fig. 14. Detection efficiency derived from the optimized selection parameters as a function of the host star magnitude.

\section{Other applications of the WTS DI light curves}

In the previous sections, we discussed the benefits of using the WTS DI light curves to detect transiting planet candidates, particularly when searching for objects with faint magnitudes $(J>16)$. The DI light curves can be used for additional analyses, such as detection and characterization of faint variable stars. In the next sections, we describe two examples of the results presented by Nefs et al. (2012) and Birkby et al. (2012), which describe the discovery of extremely-short period M-dwarf eclipsing binaries and M-dwarf eclipsing binaries (MEBs) in the WTS. We show these cases to demonstrate that DI light curves are able to improve the results reported in the literature and to provide new eclipsing binaries candidates when extending the search to fainter magnitudes.

\subsection{Extremely-short period eclipsing binaries}

Eclipsing binary stars with extremely-short periods that are below $\sim 0.22$ days are very rare systems (Rucinski 1992; Norton et al. 2011). Only a few of such objects have been discovered (e.g. Dimitrov \& Kjurkchieva 2010; Maceroni \& Montalbán 2004) so far. The parameters of these systems can put strong 
Table 9. List of new candidates around M-dwarfs detected in this work.

\begin{tabular}{|c|c|c|c|c|c|c|c|c|c|c|c|c|c|}
\hline Object & Data-set & $\overline{\bar{\alpha}}$ & $\bar{\delta}$ & $\bar{u}$ & $\bar{g}$ & $\bar{r}$ & $\bar{i}$ & $\bar{z}$ & $\overline{\bar{Z}}$ & $\bar{Y}$ & $\bar{J}$ & $\overline{\bar{H}}$ & $\overline{\bar{K}}$ \\
\hline 19b4-10711 & AP/DI & 293.0253 & 36.9168 & 24.10 & 21.85 & 20.35 & 19.07 & 18.51 & 17.99 & 17.63 & 17.17 & 16.53 & 16.25 \\
\hline $19 \mathrm{e} 3-01290$ & AP/DI & 293.0688 & 36.5510 & 26.66 & 22.68 & 21.33 & 19.94 & 19.13 & 18.71 & 18.32 & 17.75 & 17.13 & 16.87 \\
\hline $19 a 2-10046$ & AP/DI & 293.2753 & 36.3017 & 26.61 & 23.24 & 21.08 & 20.19 & 19.34 & 18.96 & 18.57 & 17.97 & 17.35 & 17.16 \\
\hline $19 \mathrm{a} 1-02980$ & AP/DI & 292.7127 & 36.3127 & 21.33 & 18.72 & 17.26 & 16.53 & 16.07 & 15.73 & 15.40 & 14.91 & 14.29 & 14.07 \\
\hline 19a1-07499 & AP/DI & 292.5977 & 36.4613 & 26.39 & 21.57 & 19.97 & 18.98 & 18.46 & 18.08 & 17.68 & 17.16 & 16.54 & 16.30 \\
\hline $19 \mathrm{e} 3-05850$ & AP/DI & 293.1396 & 36.6950 & 23.30 & 21.29 & 19.93 & 19.17 & 18.76 & 18.31 & 17.98 & 17.44 & 16.84 & 16.66 \\
\hline 19a1-10878 & AP/DI & 292.5126 & 36.4273 & 22.94 & 20.63 & 19.14 & 18.10 & 17.48 & 17.20 & 16.82 & 16.29 & 15.67 & 15.40 \\
\hline 19a1-01358 & AP/DI & 292.7526 & 36.4241 & 25.11 & 21.65 & 20.05 & 18.89 & 18.45 & 17.92 & 17.55 & 17.03 & 16.38 & 16.16 \\
\hline
\end{tabular}

Notes. The second column shows the light curve data set in which the candidates have been detected. The coordinates (J2000.0) are listed in Cols. 3 and 4 . The remaining columns provide broadband photometric measurements of our candidates in ten different filters. The $u, g, r, i, z$ $A B$-magnitudes were obtained from the SDSS and the $Z, Y, J, H, K$ magnitudes are WFCAM measurements in the Vega-system.

Table 10. Characterization of host stars for the M-dwarf sample.

\begin{tabular}{lccccccccc}
\hline \hline Object & $T_{\text {eff }}(\mathrm{K})$ & Spectral type & $\log g_{1}$ & $\log g_{2}$ & $A_{\mathrm{v}}$ & Distance $(\mathrm{pc})$ & $R 1_{\star}\left(R_{\odot}\right)$ & $R 2_{\star}\left(R_{\odot}\right)$ & $M_{\star}\left(M_{\odot}\right)$ \\
\hline $19 \mathrm{~b} 4-10711$ & 3400 & M4 & 4.94 & 4.68 & 0.02 & 676 & $0.33_{-0.09}^{+0.07}$ & $0.44_{-0.01}^{+0.01}$ & $0.34_{-0.11}^{+0.09}$ \\
$19 \mathrm{e} 3-01290$ & 3300 & M5 & 5.02 & 4.49 & 0.18 & 703 & $0.24_{-0.09}^{+0.11}$ & $0.45_{-0.02}^{+0.02}$ & $0.23_{-0.10}^{+0.11}$ \\
$19 \mathrm{a} 2-10046$ & 3500 & M3 & 4.88 & 4.50 & $0.28^{*}$ & 1554 & $0.40_{-0.07}^{+0.05}$ & $0.61_{-0.07}^{+0.02}$ & $0.43_{-0.09}^{+0.05}$ \\
$19 \mathrm{a} 1-02980$ & 3900 & M0 & 4.70 & 4.21 & 0.15 & 522 & $0.55_{-0.03}^{+0.03}$ & $0.99_{-0.05}^{+0.04}$ & $0.58_{-0.02}^{+0.04}$ \\
19a1-07499 & 3600 & M2 & 4.82 & 4.42 & 0.18 & 1063 & $0.45_{-0.05}^{+0.03}$ & $0.71_{-0.06}^{+0.04}$ & $0.48_{-0.05}^{+0.04}$ \\
19e3-05850 & 3800 & M1 & 4.77 & 3.98 & 0.01 & 1572 & $0.52_{-0.04}^{+0.03}$ & $1.26_{-0.02}^{+0.15}$ & $0.56_{-0.04}^{+0.02}$ \\
19a1-10878 & 3600 & M2 & 4.82 & 4.48 & 0.12 & 704 & $0.45_{-0.05}^{+0.03}$ & $0.66_{-0.01}^{+0.01}$ & $0.48_{-0.05}^{+0.04}$ \\
19a1-01358 & 3500 & M3 & 4.88 & 4.89 & 0.11 & 844 & $0.40_{-0.07}^{+0.05}$ & $0.39_{-0.03}^{+0.09}$ & $0.43_{-0.09}^{+0.05}$ \\
\hline
\end{tabular}

Notes. $T_{\text {eff }}$ is derived from SED-fit with the VOSA. The stellar radii $R 2_{\star}$ correspond to the best-fit of the analytic transit model (see Sect. 5.3). The distances reported in Col. 7 are estimated utilizing the extinction values found in the SED analysis, the $i$-band magnitudes reported in Table 3, and the absolute magnitudes $M_{\mathrm{i}}$, which are obtained from the isochrones.

Table 11. Comparison between the planet and binary scenarios for candidates around M-dwarfs.

\begin{tabular}{lcccccccc}
\hline \hline Object & $V$ & $\mathrm{dp}(\%)$ & $\chi_{\text {d.o.f. }}^{2}$ & $\chi_{\text {d.o.f. }}^{\prime 2}$ & $\mathrm{dp}_{1}^{\prime}(\%)$ & $\mathrm{dp}_{2}^{\prime}(\%)$ & $V_{1}^{\prime}$ & $V_{2}^{\prime}$ \\
\hline $19 \mathrm{~b} 4-10711$ & 0.69 & 23.70 & 1.7020 & 1.6952 & 23.87 & 25.90 & 0.86 & 0.66 \\
$19 \mathrm{e} 3-01290$ & 0.66 & 24.98 & 1.0759 & 1.0715 & 25.52 & 19.95 & 0.71 & 0.52 \\
$19 \mathrm{a} 2-10046$ & 0.61 & 19.63 & 2.0426 & 2.0596 & 19.52 & 17.54 & 0.62 & 0.64 \\
$19 \mathrm{a} 1-02980$ & 0.72 & 2.17 & 1.7077 & 1.6537 & 2.70 & 1.42 & 0.71 & 0.79 \\
$19 \mathrm{a} 1-07499$ & 0.68 & 7.38 & 1.3803 & 1.3759 & 7.10 & 8.01 & 0.60 & 0.90 \\
$19 \mathrm{e} 3-05850$ & 0.00 & 7.85 & 1.1237 & 1.1203 & 7.48 & 7.42 & 0.00 & 0.16 \\
$19 \mathrm{a} 1-10878$ & 0.65 & 22.70 & 1.9194 & 1.7947 & 24.19 & 19.06 & 0.65 & 0.80 \\
$19 \mathrm{a} 1-01358$ & 0.91 & 4.67 & 1.3165 & 1.3142 & 5.28 & 3.30 & 0.66 & 0.21 \\
\hline
\end{tabular}

Notes. Comparison of the eclipse shapes, eclipse depths, and $\chi_{\text {d.o.f. }}^{2}$ values of the planet scenario and binary scenario (prime values on the right side of the table).

Table 12. Characterization of candidates around M-dwarfs, according to the analytic transit fit.

\begin{tabular}{lcccccccc}
\hline \hline Candidate & \multirow{2}{*}{$t_{0}$} & $i\left(^{\circ}\right)$ & $R_{\text {planet }}\left(R_{\text {Jup }}\right)$ & $R_{\text {planet,min }}\left(R_{\text {Jup }}\right)$ & $R_{\text {planet,max }}\left(R_{\text {Jup }}\right)$ & $\chi_{\text {d.o.f. }}^{2}$ & Classification \\
\hline 19b4-10711 & 1.55274390 & 2454318.1664350 & 85.16 & 2.53 & 2.34 & 2.84 & 1.28 & $\mathrm{~B}$ \\
$19 \mathrm{e} 3-01290$ & 2.46752082 & 2454318.6477813 & 85.86 & 2.64 & 2.34 & 3.20 & 1.01 & $\mathrm{~B}$ \\
$19 \mathrm{a} 2-10046$ & 1.45677364 & 2454318.7173035 & 84.48 & 2.78 & 2.46 & 5.31 & 1.43 & $\mathrm{~B}$ \\
$19 \mathrm{a} 1-02980$ & 1.05176697 & 2454318.6516446 & 72.80 & 3.25 & 1.93 & 6.95 & 1.31 & $\mathrm{~B}$ \\
$19 \mathrm{a} 1-07499$ & 1.96038974 & 2454318.5897989 & 81.29 & 3.45 & 2.14 & 7.28 & 1.18 & $\mathrm{~B}$ \\
$19 \mathrm{e} 3-05850$ & 9.20198442 & 2454320.1712614 & 86.81 & 3.48 & 3.30 & 5.03 & 1.06 & $\mathrm{~B}$ \\
$19 \mathrm{a} 1-10878$ & 1.55498531 & 2454317.9578553 & 83.43 & 3.71 & 3.49 & 3.99 & 1.32 & $\mathrm{~B}$ \\
$19 \mathrm{a} 1-01358$ & 1.10712079 & 2454318.6005745 & 76.76 & 4.94 & 1.71 & 7.12 & 1.09 & $\mathrm{~B}$ \\
\hline
\end{tabular}

Notes. Orbital and planetary parameters derived from the analytic transit model fit. All candidates are too large and are most likely transiting brown dwarfs or low-mass stars. 
Table 13. List of extremely-short period M-dwarf eclipsing binary systems found in this work.

\begin{tabular}{|c|c|c|c|c|c|c|c|c|c|c|c|c|c|c|c|}
\hline Object & $\alpha$ & $\delta$ & Period (days) & $t_{0}$ & $\mathrm{dp}_{2}^{\prime} / \mathrm{dp}_{1}^{\prime}$ & $J$ & $u$ & $g$ & $r$ & $i$ & $z$ & $(r-i)$ & $(i-z$ & $\operatorname{rms}(\mathrm{AP})$ & $\operatorname{rms}(\mathrm{DI})$ \\
\hline $19 \mathrm{c} 3-12753$ & 294.3839 & 36.9062 & 0.1859752355 & 2454317.8449795 & 1.56 & 17.87 & 24.93 & 22.80 & 21.09 & 20.08 & 19.17 & 1.01 & 0.91 & 0.041 & 0.028 \\
\hline $19 \mathrm{~b} 2-04235$ & 93.3342 & 36.4255 & 0.1974392134 & 2454317.9485581 & 1.52 & 17.32 & 22.68 & 22.07 & 20.16 & 19.43 & 18.77 & 0.74 & 0.66 & 0.027 & 0.023 \\
\hline $19 \mathrm{c} 2-10801$ & 294.2404 & 36.3471 & 0.1977343597 & 2454317.9436689 & 0.88 & 17.78 & 25.86 & 21.37 & 20.24 & 19.66 & 19.36 & 0.59 & 0.29 & & 0.049 \\
\hline $19 \mathrm{e} 3-11606$ & 293.2310 & 36.6396 & 0.2106563732 & 2454317.7894282 & 1.45 & 17.97 & 25.01 & 21.71 & 20.31 & 19.67 & 19.40 & 0.65 & 0.26 & 0.062 & 0.050 \\
\hline $19 \mathrm{c} 1-00478$ & 293.8732 & 36.4661 & 0.2261831592 & 2454317.8755733 & 1.57 & 17.18 & 23.53 & 20.44 & 19.40 & 18.70 & 18.35 & 0.69 & 0.36 & 0.028 & 0.022 \\
\hline
\end{tabular}

Notes. Extremely-short period M-dwarf eclipsing binary systems with periods below 0.23 days. We list the period, epoch, eclipse depth ratio, the WFCAM $J$-band, and SDSS ugriz photometry for each candidate. The last two columns provide information on the $4 \sigma$ clipped rms of the light curves after removing the periodic signal. In general, the precision of the DI light curves is significantly better than the precision of the AP light curves. This is due to the fact that all objects are fainter than 17 mag in the $J$-band, which is in the regime where DI light curves present an improvement over AP light curves (see Sect. 3).

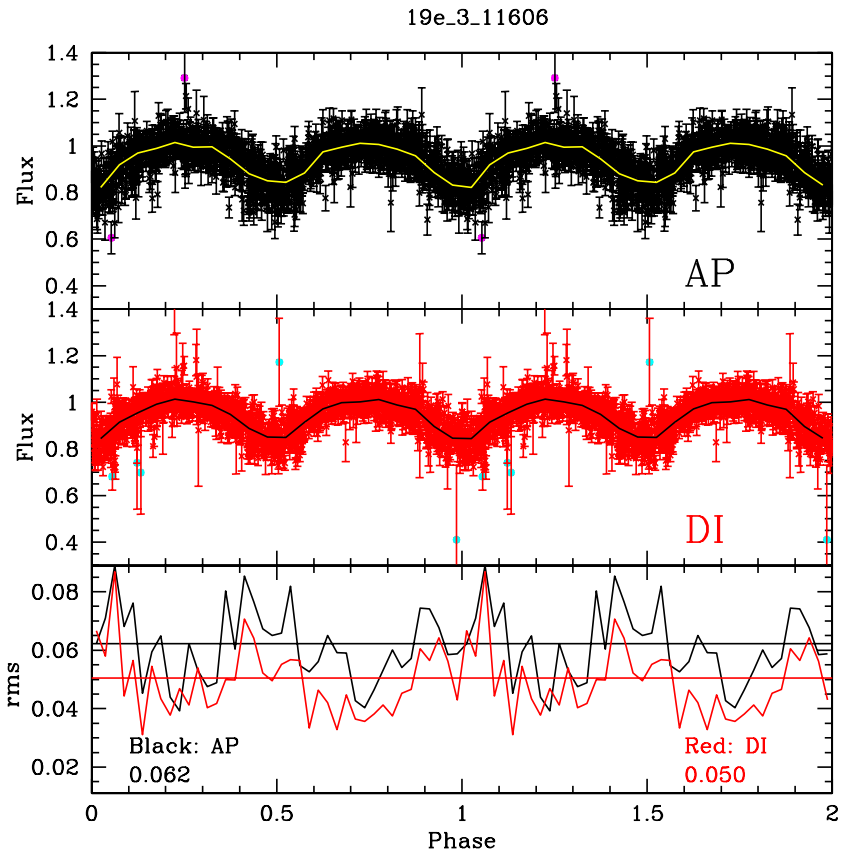

Fig. 15. Comparison between the AP and DI light curves of an extremely-short period M-dwarf eclipsing binary system (19e-3-11606) found in the DI light curves. Black and red points in the upper two panels correspond to the AP and DI light curves, respectively. The yellow and black solid lines connect the median values in 40 bins with a size of 0.025 phase units. The lower panel shows the $4 \sigma$ clipped rms of the residuals in each bin after subtracting the median. The horizontal lines represent the average rms of the residuals, which are 0.062 and 0.050 for the AP and DI light curves, respectively.

constraints on formation and evolution theories of low-mass stars (Devor 2005; Derekas et al. 2007). Recently, Nefs et al. (2012) reported a sample of 31 eclipsing binaries with periods smaller than 0.3 days that were found in the WTS 03, 07, 17 and $19 \mathrm{~h}$ fields. Four of them are M-dwarf binaries with orbital periods that are considerably shorter than the sharp cutoff period of $\sim 0.22$ days. We ran our detection algorithm on the DI light curves using the same input parameters reported in Nefs et al. (2012). We reproduce periods and $t_{0}$ values of the objects reported previously. In addition, we detected five new eclipsing binaries with periods shorter than 0.23 days. All systems satisfy the color cuts and fit with the red sample (i.e. M-dwarfs) presented in Nefs et al. (2012). We additionally use the SDSS color criteria from Ivezić et al. (2005) to eliminate the possibility of being in presence of RR Lyrae. Another cases of false positives are caused by contamination effects from nearby stars and stellar variability produced by star spots. However, we reject both scenarios, since DI method is designed to reduce the effects caused by very near stellar neighbors. The phase folded light curves also do not present a large scatter in their amplitude, which is generally an indication of variability generated by star spots. Table 13 lists the parameters of these systems. Note that the periodic signal of 19c-2-10801 could not be found in the AP light curve at all. We checked for a mismatch in the cross-identification procedure but could not find any object in the vicinity with a comparable variability. We show the folded light curves of all five objects in Fig. 18.

To show the improvement in the precision of the DI light curves at faint magnitudes, we carry out a statistical comparison of the AP and DI light curves for the system 19e-3-11606, which has a brightness of $J=17.97 \mathrm{mag}$. Figure 15 shows the phase-folded DI and AP light curves and the rms with respect to the mean in 40 equally spaced bins. The horizontal lines show the $4 \sigma$ clipped rms for both light curves, which are 0.050 and 0.062 , respectively. The DI light curve, therefore, has an rms that is about $12 \mathrm{mmag}$ lower, which is a little bit less than what we expected for an object with $J=18.0 \mathrm{mag}$. Looking at the other four detected objects, we find that this is a general trend. The lower difference in rms can be explained by in that we are looking at variable objects for which the sysrem algorithm cannot reduce systematic effects in an efficient way. It seems that the AP light curves are less affected by this than the DI light curves.

\subsection{M-dwarf eclipsing binaries}

Recently, Birkby et al. (2012) reported the detection of 16 M-dwarf eclipsing binary systems (MEBs) with $J<16$ mag found in the WTS AP light curves. These systems are particularly interesting because they provide important information about the fundamental properties of the most abundant stars in our Galaxy (Henry et al. 1997). Nevertheless, the existing theoretical models that describe the evolution of low-mass stars differ from the observed properties of M-dwarfs (López-Morales $\&$ Ribas 2005). More observations and characterization of MEBs can provide new evidence to develop better and more accurate low-mass stellar evolution models (Birkby et al. 2012). We investigate the potential of extending the search for MEBs to fainter systems with magnitudes $J \leq 18$ mag, making use of the improvement in the photometric precision of the DI light curves. In Table 12, we report eight candidates classified as eclipsing binary systems, where the objects 19a1-02980 and 19a1-10878 show strong evidence of being MEBs. The system 19a1-02980 was actually reported and confirmed as MEB in Birkby et al. (2012), which supports the remaining fainter detections, since 


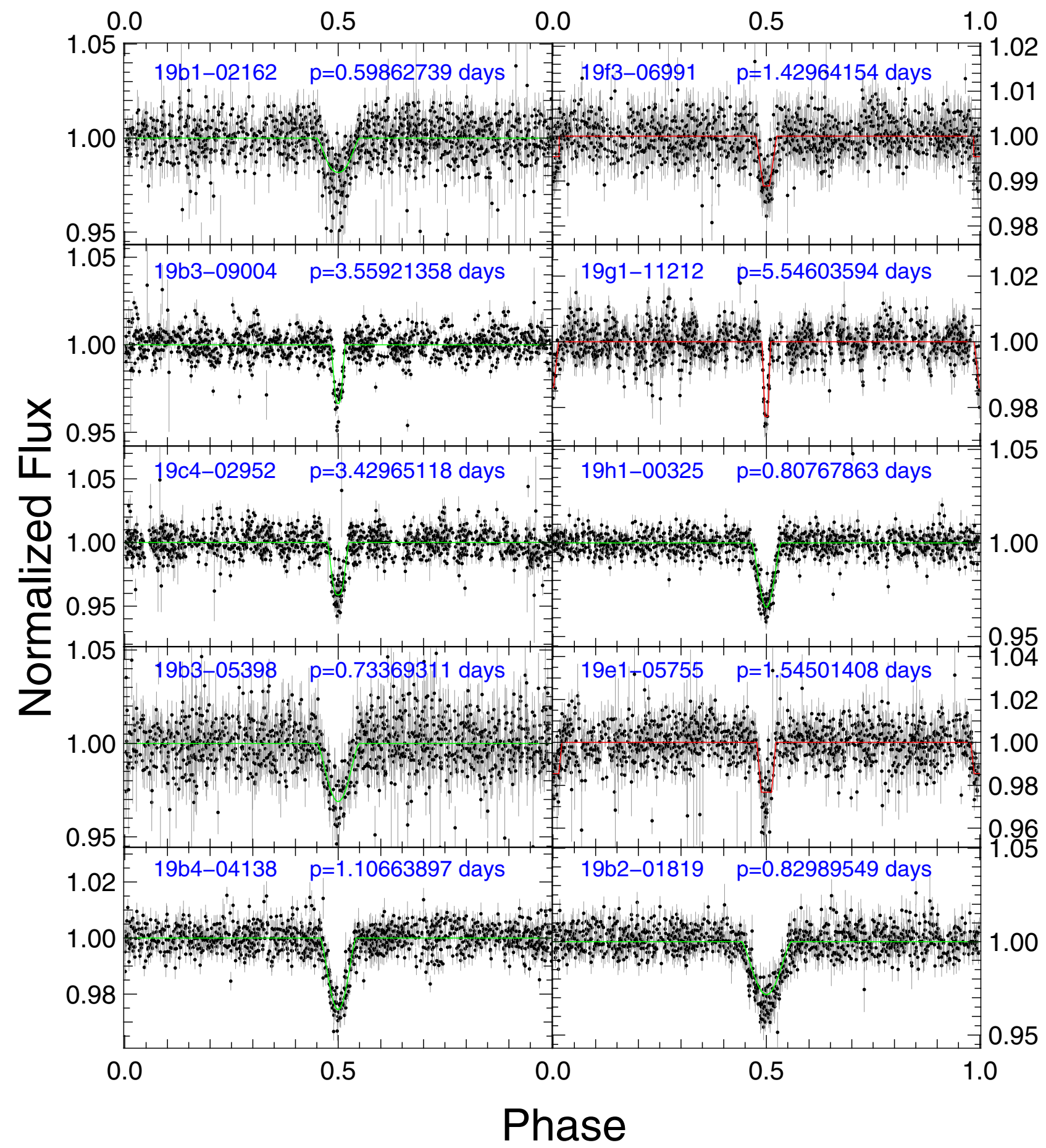

Fig. 16. Phase-folded light curves of the ten candidates around F-G-K stars found in the WTS $19 \mathrm{~h}$ field. We overplot the best-fitting single eclipse transit models (green lines) for all objects for which the binary fit does not show an improvement over the single eclipse. The light curves with two eclipses are shown together with the best-fitting trapeziod model (red lines).

they were identified through the same process. Furthermore, we found a third system (19c4-06354) with similar characteristics as the two candidates mentioned above by an additional analysis carried out on the AP and DI light curves, so we also classify this object as a MEB candidate. In Fig. 17, we show the folded light curve of the three MEB candidates. The parameters associated with the main stellar companion of 19a1-02980 and 19a1-10878 are listed in Table 10. For the candidate 19c4-06354, we report a primary stellar companion with $J$-band $=17.97$ in a short period system of $P \sim 0.76$ days and low $T_{\text {eff }}$ of $3500 \mathrm{~K}$. Due to the faint magnitude of the primary stellar companion, this MEB candidate was found only in the DI light curves. A more extensive and meticulous search for MEB systems in the DI light curves (which is out of the scope of this work) could potentially reveal many more detections in the future.

\section{Conclusions}

We carried out a quantitative comparison between the photometric precision of two different sets of light curves from the $19 \mathrm{~h}$ field, which represents the most complete field of the WTS. The light curves were obtained using two different photometric 


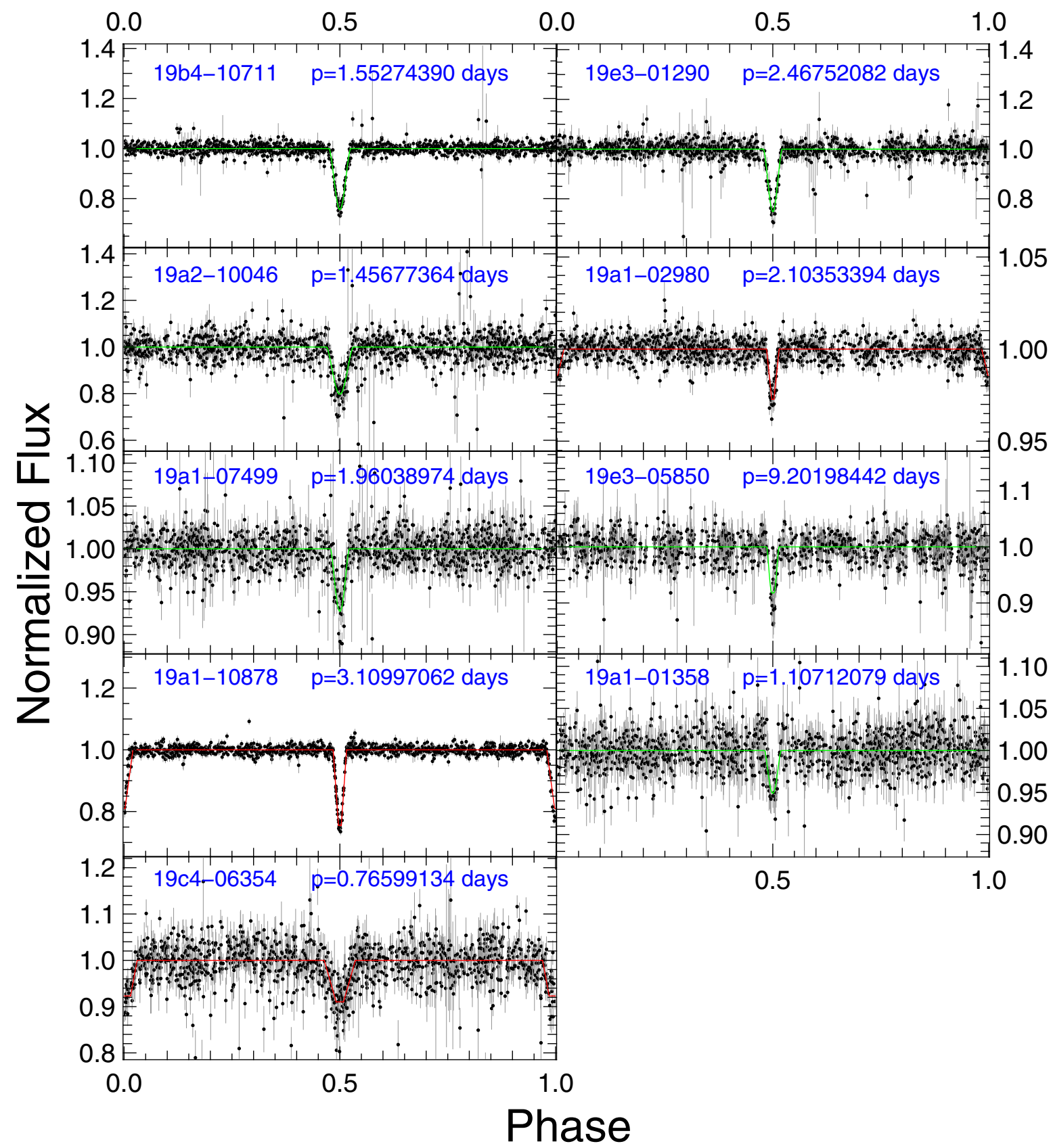

Fig. 17. Phase-folded light curves of nine candidates that orbit M-dwarfs from the WTS $19 \mathrm{~h}$ field. As it was done for the candidates around F-G-K-stars, we overplot the best-fitting single eclipse with green solid lines, whereas the light curves with two eclipses are shown with the bestfitting trapeziod model, using red-solid lines. The objects with two eclipses are reported in Sect. 7.2 as MEB candidates. Objects 19a1-02980 and 19a1-10878 were found by our selection criteria during the process of transiting planet detection, whereas the system 19c4-06354 was separately detected by an additional analysis carried out on the AP and DI light curves.

techniques, AP and DI. The sysrem algorithm was used to remove systematic effects in both data sets and corrected the light curves by scaling the error bars. The WTS AP light curves reach a slightly better photometric precision (by $\sim 1 \mathrm{mmag}$ ) than the DI light curves for objects brighter than $J \approx 15.5$ mag. On the other hand, the DI light curves show a significant improvement of $\sim 2-20$ mmag for sources with magnitudes larger than $J=16$ mag.

A modified version of the box-fitting algorithm was employed to search for transiting planets in the survey. Our algorithm uses the standard BLS to searches for the best trial period and subsequently makes a trapezoid re fit to the folded light curve, providing a new estimation of the transit depth.
A $\chi^{2}$ comparison shows that the new trapezoid fit provides better results than the traditional box-fitting model. The algorithm also calculates a new parameter based on the geometry of the new trapezoid fit, the $V$-shape parameter. This parameter has proven to be very efficient in the identification and removal of eclipsing binaries from the candidate sample.

To select our candidates, we proposed a set of selection criteria; six of them are based on the experience of previous works. Additionally, two new criteria were incorporated, which take advantage of the results obtained with our transit detection algorithm, such as the $V$-shape parameter. The set of parameters of our selection criteria was optimized using Monte Carlo simulations by injecting transit signals to both the 

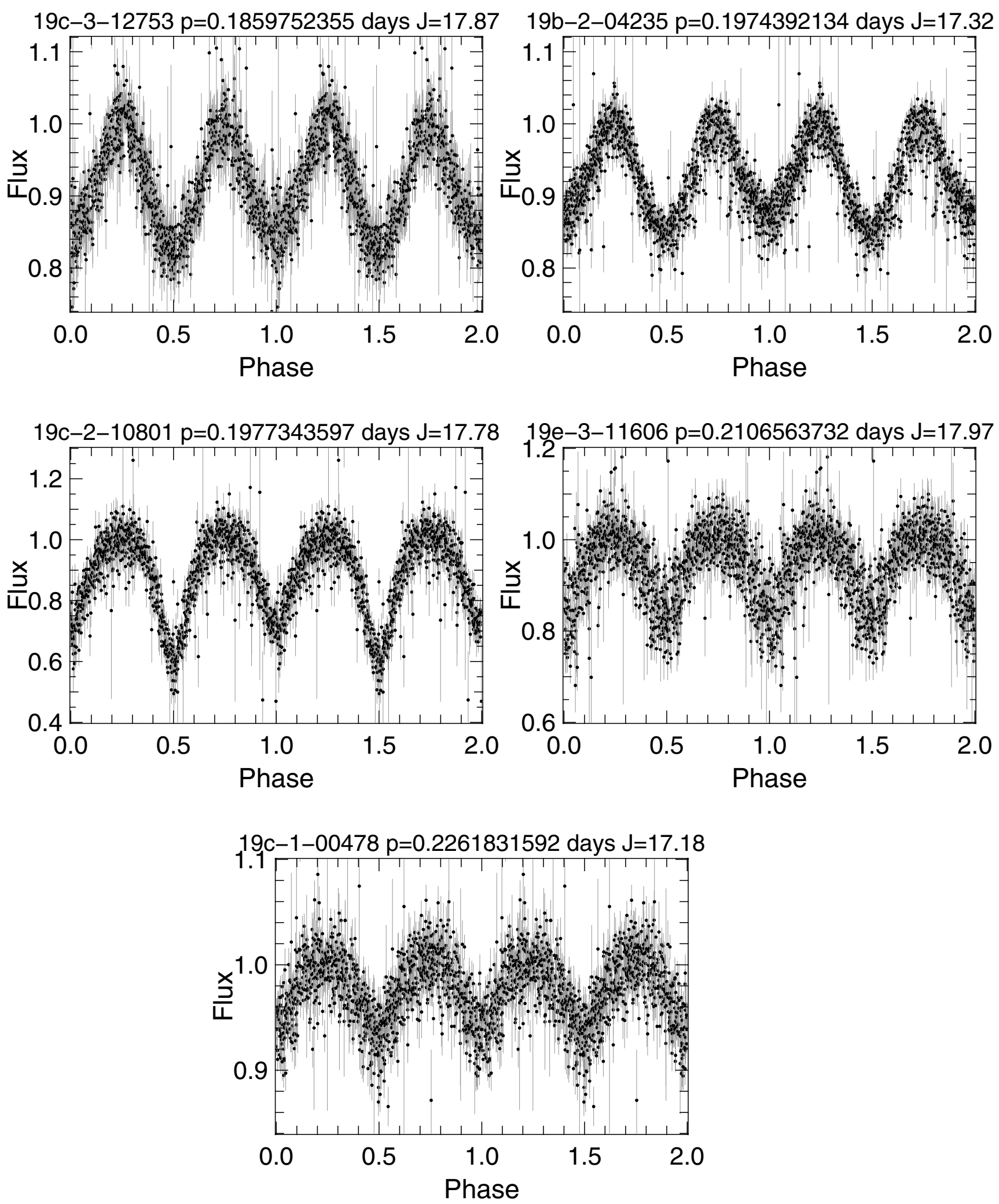

Fig. 18. Phase-folded light curves of the five extremely-short period eclipsing binaries found in this work.

AP and DI light curves. The light curves were split in two different sets, one for F-G-K-stars, and a second for M-dwarfs. The optimization of the criteria was performed in both sets separately. The selection criteria have shown the capability of detecting 200 candidates in the DI and AP light curves from a original sample of $\sim 475000 \mathrm{~F}-\mathrm{G}-\mathrm{K}$-stars, while 196 candidates were detected in both sets of light curves from the M-dwarfs sample.
We carried out a visual examination on the detections and identified 18 relevant transit planet candidates.

A detailed analysis of the 18 candidates was conducted to distinguish planetary from binary candidates, which provides physical parameters of the candidates and their host stars. The analysis includes a characterization of the parent star and a transit fit of the light curve using a realistic model proposed by 
Mandel \& Agol (2002). Furthermore, we performed a secondary eclipse fit to the phase folded light curve using the double period to detect potential differences in the $\chi_{\text {d.o.f. }}^{2}$ and/or the depths of the primary and secondary eclipses that could be an indication of an eclipsing binary system. In our analysis, only one object is classified as a planet candidate, which is proposed for photometric follow-up. The remaining 17 candidates have large bestfitting radii and are therefore classified as binary candidates.

No planet candidates orbiting an M-dwarf was found; therefore, the null detection presented in Kovács et al. (2013) was confirmed. A detailed sensitivity analysis allowed us to derive an upper limit on the occurrence rate of giant planets around M-dwarfs with periods below 10 days. Increasing the number of target stars by going one magnitude deeper, we were able to set a $95 \%$ confidence upper limit of $1.1 \%$, which is significantly lower than any limit published so far. Another applications of the WTS DI light curves were reported. We presented the detection of five new ultra-short period eclipsing binaries with periods below 0.23 days and $J>17$ mag. In addition, three detached M-dwarf eclipsing binary candidates were reported; two of them were found in both the AP and DI light curves, while the third and faintest candidate was only detected in the DI light curves sample. These results show that the DI light curves are able to reproduce and improve results reported in the literature. In conclusion, the WTS DI light curves are useful for many purposes, such as detection of transit planet candidates and rare eclipsing binary systems, especially when pushing the limits to fainter magnitudes.

Acknowledgements. We acknowledge the support of RoPACS network during this research, a Marie Curie Initial Training Network funded by the European Commission's Seventh Framework Programme. This publication makes use of the VOSA, developed under the Spanish Virtual Observatory project supported from the Spanish MICINN through grant AyA2008-02156. This research has been also funded by Spanish grants AYA2012-38897-C02-01, PRICIT-S2009/ESP-1496 and AyA2011-30147-C03-03. This work has made use of the NASA/IPAC Extragalactic Database (NED) which is operated by the Jet Propulsion Laboratory, California Institute of Technology, under contract with the National Aeronautics and Space Administration. Furthermore, we have made use of NASA's Astrophysics Data System, and the SIMBAD database operated at the CDS, Strasbourg, France.

\section{References}

Adelman-McCarthy, J. K., et al. 2009, VizieR Online Data Catalog, II, 294

Aigrain, S., Barge, P., Deleuil, M., et al. 2008, in 14th Cambridge Workshop on Cool Stars, Stellar Systems, and the Sun, ed. G. van Belle, ASP Conf. Ser., 384,270

Alard, C., \& Lupton, R. H. 1998, ApJ, 503, 325

Baraffe, I., Chabrier, G., Allard, F., \& Hauschildt, P. H. 1998, A\&A, 337, 403

Barge, P., Baglin, A., Auvergne, M., \& CoRoT Team. 2008, in IAU Symp. 249, eds. Y.-S. Sun, S. Ferraz-Mello, \& J.-L. Zhou, 3

Bayo, A., Rodrigo, C., Barrado, Y., Navascués, D., et al. 2008, A\&A, 492, 277

Berta, Z. K., Irwin, J., Charbonneau, D., Burke, C. J., \& Falco, E. E. 2012, AJ, 144,145
Birkby, J., Nefs, B., Hodgkin, S., et al. 2012, MNRAS, 426, 1507

Birkby, J. L., Cappetta, M., Cruz, P., et al. 2013a, in European Physical Journal Web of Conferences, 47, 1004

Birkby, J. L., Cappetta, M., Cruz, P., et al. 2013b, MNRAS, submitted

Borucki, W. J., Koch, D., Basri, G., et al. 2010, Science, 327, 977

Burke, C. J., Gaudi, B. S., DePoy, D. L., \& Pogge, R. W. 2006, AJ, 132, 210

Cappetta, M., Saglia, R. P., Birkby, J. L., et al. 2012, MNRAS, 427, 1877

Castelli, F., Gratton, R. G., \& Kurucz, R. L. 1997, A\&A, 318, 841

Charbonneau, D., Brown, T. M., Latham, D. W., \& Mayor, M. 2000, ApJ, 529, L45

Claret, A., \& Bloemen, S. 2011, A\&A, 529, A75

Defaÿ, C., Deleuil, M., \& Barge, P. 2001, A\&A, 365, 330

Derekas, A., Kiss, L. L., \& Bedding, T. R. 2007, ApJ, 663, 249

Devor, J. 2005, ApJ, 628, 411

Dimitrov, D. P., \& Kjurkchieva, D. P. 2010, MNRAS, 406, 2559

Dotter, A., Chaboyer, B., Jevremović, D., et al. 2008, ApJS, 178, 89

Giacobbe, P., Damasso, M., Sozzetti, A., et al. 2012, MNRAS, 424, 3101

Gould, A., Dorsher, S., Gaudi, B. S., \& Udalski, A. 2006, Acta Astron., 56, 1

Hartman, J. D., Gaudi, B. S., Holman, M. J., et al. 2009, ApJ, 695, 336

Henry, T. J., Ianna, P. A., Kirkpatrick, J. D., \& Jahreiss, H. 1997, AJ, 114, 388

Henry, G. W., Marcy, G. W., Butler, R. P., \& Vogt, S. S. 2000, ApJ, 529, L41

Hodgkin, S. T., Irwin, M. J., Hewett, P. C., \& Warren, S. J. 2009, MNRAS, 394, 675

Howard, A. W., Marcy, G. W., Bryson, S. T., et al. 2012, ApJS, 201, 15

Irwin, M. J. 1985, MNRAS, 214, 575

Irwin, M., \& Lewis, J. 2001, New Astron. Rev., 45, 105

Irwin, J., Irwin, M., Aigrain, S., et al. 2007, MNRAS, 375, 1449

Irwin, J., Charbonneau, D., Nutzman, P., \& Falco, E. 2009, in 15th Cambridge Workshop on Cool Stars, Stellar Systems, and the Sun, ed. E. Stempels, AIP Conf. Proc., 1094, 445

Ivezić, Ž., Vivas, A. K., Lupton, R. H., \& Zinn, R. 2005, AJ, 129, 1096

Jehin, E., Gillon, M., Queloz, D., et al. 2011, The Messenger, 145, 2

Jenkins, J. M., Chandrasekaran, H., McCauliff, S. D., et al. 2010, in Proc. SPIE, 7740, 0D

Kaltenegger, L., \& Traub, W. A. 2009, ApJ, 698, 519

Kasting, J. F., Whitmire, D. P., \& Reynolds, R. T. 1993, Icarus, 101, 108

Kleinmann, S. G., Lysaght, M. G., Pughe, W. L., et al. 1994, Ap\&SS, 217, 11

Koppenhoefer, J., Afonso, C., Saglia, R. P., \& Henning, T. 2009, A\&A, 494, 707

Kovács, G., Zucker, S., \& Mazeh, T. 2002, A\&A, 391, 369

Kovács, G., Hodgkin, S., Sipőcz, B., et al. 2013, MNRAS, 433, 889

Law, N. M., Kraus, A. L., Street, R., et al. 2012, ApJ, 757, 133

López-Morales, M., \& Ribas, I. 2005, ApJ, 631, 1120

Maceroni, C., \& Montalbán, J. 2004, A\&A, 426, 577

Mandel, K., \& Agol, E. 2002, ApJ, 580, L171

Mayor, M., \& Queloz, D. 1995, Nature, 378, 355

Mazeh, T., Guterman, P., Aigrain, S., et al. 2009, A\&A, 506, 431

Meeus, J. 1982, Astronomical formulae for calculators, 2nd edn. (Richmond, VA), 43

Montalto, M., Piotto, G., Desidera, S., et al. 2007, A\&A, 470, 1137

Nefs, S. V., Birkby, J. L., Snellen, I. A. G., et al. 2012, MNRAS, 425, 950

Norton, A. J., Payne, S. G., Evans, T., et al. 2011, A\&A, 528, A90

Pietrukowicz, P., Minniti, D., Díaz, R. F., et al. 2010, A\&A, 509, A4

Pont, F., Zucker, S., \& Queloz, D. 2006, MNRAS, 373, 231

Robin, A. C., Reylé, C., Derrière, S., \& Picaud, S. 2003, A\&A, 409, 523

Rucinski, S. M. 1992, AJ, 103, 960

Scalo, J., Kaltenegger, L., Segura, A. G., et al. 2007, Astrobiology, 7, 85

Snellen, I. A. G., van der Burg, R. F. J., de Hoon, M. D. J., \& Vuijsje, F. N. 2007, A\&A, 476, 1357

Tamuz, O., Mazeh, T., \& Zucker, S. 2005, MNRAS, 356, 1466

Tarter, J. C., Backus, P. R., Mancinelli, R. L., et al. 2007, Astrobiology, 7, 30

Tomaney, A. B., \& Crotts, A. P. S. 1996, AJ, 112, 2872 